\title{
Transformation of Satellite Breakup Distribution for Probabilistic Orbital Collision Hazard Analysis
}

\author{
Stefan Frey* and Camilla Colombo⿱亠 \\ Polytechnic University of Milan, 20156 Milan, Italy \\ https://doi.org/10.2514/1.G004939
}

\begin{abstract}
Fragmentation clouds from explosions or collision of payloads and rocket bodies in space pose a threat to objects in Earth orbit. Most of the fragments are too small to be tracked and can only be accounted for statistically. Here, a framework for the fully statistical treatment of a fragmentation cloud, its evolution and ramifications, without the need of simplifying assumptions, is presented. The cloud is modeled as an uncertainty around a single fragment, which can be propagated using any of the existing, nondeterministic, nonlinear orbital uncertainty propagation methods. This work is focused on providing the initial distribution and the estimation of the statistical collision probability. The NASA standard breakup model is revisited to derive a probability distribution of the initial fragment cloud. Two density transformation methods are discussed to obtain the distribution in a subset of orbital elements, suitable for mid- to long-term evolution. The fragment spatial density and the impact rates on targets in any orbit are obtained. The method is applied to show the fragment cloud distribution of a payload collision in low Earth orbit (LEO). Its collision probability with a satellite in LEO and a rocket body in the geostationary transfer orbit are estimated. The result is compared against, and shows the limitations of, sampling and methods based on finite differences.
\end{abstract}

\section{Nomenclature}

$A$ $A / m$

$a$

$e$

$f$

$h$

$\mathcal{I}$

$i$

$\boldsymbol{J}$

$m$

$N$

$n_{x}$

$p$

$p_{x}$

$R$

$r, r$

$s$

$\mathcal{T}$

$u$

$\boldsymbol{v}, v$

$\boldsymbol{x}$

$\alpha$

$\Delta \boldsymbol{v}, \Delta v$

$\eta$

$\mu$

$\Omega$

$\omega$

cross-sectional area, $\mathrm{m}^{2}$

area-to-mass ratio, $\mathrm{m}^{2} / \mathrm{kg}$

semimajor axis, $\mathrm{m}$ or $\mathrm{km}$

eccentricity

true anomaly, rad or deg

inertial frame

inclination, rad or deg

Jacobian

mass, $\mathrm{kg}$

number of fragments

semiparameter, $\mathrm{m}$ or $\mathrm{km}$

satellite frame

phase space

Keplerian elements

number of impacts specific angular momentum, $\mathrm{m}^{2} / \mathrm{s}$ or $\mathrm{km}^{2} / \mathrm{s}$

fragment phase space density function in $\boldsymbol{x}$

probability density function in $\boldsymbol{x}$

central planet radius, $\mathrm{m}$ or $\mathrm{km}$

orbital radius and magnitude, $\mathrm{m}$ or $\mathrm{km}$

state in Cartesian coordinates

argument of latitude, rad or deg

orbital velocity and magnitude, $\mathrm{m} / \mathrm{s}$ or $\mathrm{km} / \mathrm{s}$

orbital velocity impulse and magnitude, $\mathrm{m} / \mathrm{s}$ or $\mathrm{km} / \mathrm{s}$

gravitational parameter, $\mathrm{m}^{3} / \mathrm{s}^{2}$ or $\mathrm{km}^{3} / \mathrm{s}^{2}$

right ascension of ascending node, $\mathrm{rad}$ or $\mathrm{deg}$

argument of perigee, rad or deg

\section{Introduction}

$\mathbf{F}$ OR many space applications, uncertainties around states in general and velocity changes in particular need to be considered. In spacecraft propulsion, the thrust impulse and direction are afflicted

Received 5 November 2019; revision received 30 June 2020; accepted for publication 7 July 2020; published online Open Access 9 September 2020. Copyright $\left({ }^{\circ} 2020\right.$ by Stefan Frey and Camilla Colombo. Published by the American Institute of Aeronautics and Astronautics, Inc., with permission. All requests for copying and permission to reprint should be submitted to $\mathrm{CCC}$ at www.copyright.com; employ the eISSN 1533-3884 to initiate your request. See also AIAA Rights and Permissions www.aiaa.org/randp.

*Ph.D. Student, Department of Aerospace Science and Technology, Via La Masa, 34; stefan.frey@ polimi.it.

Associate Professor, Department of Aerospace Science and Technology, Via La Masa, 34; camilla.colombo@polimi.it. with uncertainty [1]. Initial orbit determination deals with uncertainty in observations [2]. The impact probability used for the verification of compliance with the planetary protection requirements is a function of state uncertainties [3]. In asteroid deflection, the impulse imparted onto the asteroid comes with uncertainties in magnitude and direction [4]. The distribution of a cloud of fragments, or fragment continuum, stemming from an explosion or collision can equally be treated as an uncertainty, although a large one, around the parent object. The first to publish a continuum approach in the context of space debris was Heard in 1976 [5], modeling the fragment distribution originating from an exploding satellite as a continuum of noninteracting particles. Considering linearized two-body dynamics for the short-term evolution of fragments on circular orbits, he solves the Liouville's equation of the corresponding Hamiltonian system with the method of characteristics.

Jehn [6] extends the approach for estimation of the short- to midterm evolution through consideration of an extended phased approach proposed by McKnight and Lorenzen [7]. The phased approach separates the evolution of the cloud into different phases. Right after the fragmentation, i.e., over the short-term, the particles remain close to each other forming an ellipse. Because of differences in energy, some fragments revolve faster than others. Over the midterm, the fragments randomize in the mean anomaly, forming a torus along the parent orbit and permitting the modeling of the cloud independently of the anomaly. The various precession rates of the node and line of apsides, caused by the nonspherical central body, dissemble the torus over the long-term through randomization in those two elements until finally the cloud forms a band around Earth, limited in latitude by the parent inclination.

The long-term evolution of space debris and fragmentation clouds can be solved analytically using the method of characteristics, if circular orbits only are considered [8,9]. However, the solution requires further assumptions on the dynamics, such as a simple atmospheric drag model. Alternatively, the solution can be propagated numerically, however, requiring increasing complexity to obtain the density across the full domain $[10,11]$. Instead of finding the solution of the continuity along the characteristic curves, the solution can be approximated via discretization of the phase space. Within each bin the Liouville equation is (using finite differencing) replaced by its discretized version and solved numerically [12-15]. Such methods become computationally infeasible if the dimensionality of the phase space exceeds three; hence they are not applicable to short- to midterm problems.

The collision probability, probabilistic in nature owing to the many uncertainties present, can naturally be derived from a statistical definition of the fragment distribution. Kessler and Cour-Palais [16] applied the method developed by Öpik [17] for the estimation of the 
collision rate between satellites through their spatial density. A quick review of the method is given in Appendix A. A similar statistical and geometrical approach is applied to calculate the collision probability of the background population or a cloud of fragments with a satellite flying through the particles [21-23]. Tools based on probabilistic methods are computationally efficient; however, they suffer from restricting assumptions and limitations on the force model, orbital geometries, or dimensionality.

Today, the engineering tools to estimate the evolution of space debris, and its effect on resident space objects in terms of collision probability, are predominately based on deterministic techniques. Through sampling of the NASA standard breakup model (SBM) [24], fragments are drawn and propagated individually. Statistical results are obtained through repeated Monte Carlo simulations. Deterministic tools, such as NASA's LEGEND [25] or European Space Agency's DELTA [26], have prevailed over probabilistic techniques because of their ease of use and flexibility. They can consider any force model, any orbital region, and any dimensionality and are extendable for various source and sink terms, such as active debris removal. However, the propagation of many fragments is computationally expensive.

To efficiently calculate the collision probability using deterministic models, the CUBE algorithm was developed [27], following the work of Kessler [18]. The name comes from the discretization of the physical space into spatial cubes. Instead of randomizing the node, line of apsides, and anomaly term, the method randomly samples the individual instances in time, i.e., in mean anomaly. Whenever two objects end up in the same cube, the number of collisions is estimated according to Eq. (A4). Doing so, the complexity of the problem rises only linearly in the number of objects, rather than quadratically. However, the collision probability scales with the cube size [28], a nonphysical consequence of the estimation method. To be accurate the cubes need to be small and the sampling intervals short, further slowing down deterministic models. A further problem of deterministic methods is that they lack the sensitivity to well describe low probabilistic events [29]. As such, they fail to give a good approximation of the effects of a fragmentation on a single mission.

In recent years, the topic of nondeterministic, nonlinear orbital uncertainty propagation received increased attention [30]. It has the potential to eliminate the assumptions and limitations of the probabilistic techniques for debris propagation introduced above, while keeping the computational effort low [11]. Additionally, the application of the Dirac delta function to calculate the spatial density of a single object given a statistical description in orbital elements enables the evaluation of the collision probability without any hypothesis on the orbit geometry [31,32].

This work aids the development of a fully probabilistic fragmentation cloud evolution model for estimation of the collision hazard. Rather than introducing a method for the propagation of the continuum (or uncertainty) itself, it provides the framework to do so. The transformations of any density distribution given in Cartesian coordinates to obtain densities in the frame suitable for mid- to longterm propagation, or vice versa for the derivation of the collision probability, are introduced in Sec. II. A probabilistic formulation of the initial fragment density distribution, based on the NASA SBM, is derived in Sec. III. A novel technique to calculate the fragment spatial density and number of impacts directly from the density distribution given in orbital elements (with only mild assumptions) is presented in Sec. IV. Instead of considering the statistical distribution of the target object, the technique evaluates the collision probability using the density distribution of the fragment cloud. The application of the technique to the case of a fragmentation from a payload collision is illustrated and compared against sampling in terms of accuracy in

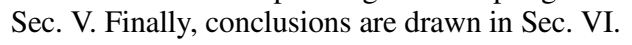

\section{Transformations}

\section{A. Preliminary Notes}

Uncertainties are described using a probability density function, $p_{x}=p(\boldsymbol{x})$, in the phase space $\boldsymbol{x} \in \mathbb{R}^{m}$, which by definition is required to sum to unity if integrated over $x$, i.e.,

$$
\int_{\mathbb{R}^{m}} p_{x} \mathrm{~d}^{m} \boldsymbol{x}=1
$$

Here, $p_{x}$ describes the probability to find a single fragment at any location in $\boldsymbol{x}$, where $\boldsymbol{x}$ can contain the position and velocity of a fragment as well as its physical characteristics. Instead, the phase space density $n_{x}=n(\boldsymbol{x})$ describes the number of fragments present in an infinitesimal volume around $\boldsymbol{x}$. Integration of $n_{x}$ over the full domain yields the total number of fragments, $N$, i.e.,

$$
\int_{\mathbb{R}^{m}} n_{x} \mathrm{~d}^{m} \boldsymbol{x}=N
$$

The fragment probability density function and the phase space density function differ only in the normalization constant. Hence, the phase space density function can be treated as a probability density function.

For the derivation of the transformation, two different Cartesian frames are required. The inertial frame $\mathcal{I}=\{\hat{\boldsymbol{x}}, \hat{\boldsymbol{y}}, \hat{z}\}$ is centered at the main attracting body and is independent of the orbit configuration. The Keplerian elements $\boldsymbol{\alpha}=(\text { a e } i \Omega \omega f)^{T}$, consisting of semimajor axis $a$; eccentricity $e$; inclination $i$; right ascension of ascending node, $\Omega$; argument of perigee, $\omega$; and true anomaly $f$, are defined with respect to $\mathcal{I}$. The elements in $\boldsymbol{\alpha}$ can be found from a state in Cartesian coordinates described in any frame, $s=\left(\boldsymbol{r}^{T} \boldsymbol{v}^{T}\right)^{T}$, with the orbital radial vector $\boldsymbol{r}$ and the orbital velocity vector $\boldsymbol{v}$. The function relating the two sets, $\alpha=\varphi_{\alpha s}(s)$, can be found in literature (e.g., [33] Chap. 2.6)

The satellite frame $\mathcal{T}=\{\hat{\boldsymbol{t}}, \hat{\boldsymbol{n}}, \hat{\boldsymbol{h}}\}$ is centered at the orbiting body, with its primary $\hat{\boldsymbol{t}}$ axis aligned with $\boldsymbol{v}$. The tertiary $\hat{\boldsymbol{h}}$ axis is aligned with the orbit normal, and the secondary $\hat{\boldsymbol{n}}$ axis, lying in the orbital plane pointing inward, completes the coordinate system. Thus, the rotation matrix from $\mathcal{T}$ to $\mathcal{I}$ is defined as

$$
\boldsymbol{A}_{\mathcal{I T}}=\left[\begin{array}{lll}
\hat{\boldsymbol{t}}_{\mathcal{I}} & \hat{\boldsymbol{n}}_{\mathcal{I}} & \hat{\boldsymbol{h}}_{\mathcal{I}}
\end{array}\right]
$$

with

$$
\hat{\boldsymbol{t}}_{\mathcal{I}}=\frac{\boldsymbol{v}_{\mathcal{I}}}{\|\boldsymbol{v}\|} \quad \hat{\boldsymbol{h}}_{\mathcal{I}}=\frac{\boldsymbol{r}_{\mathcal{I}} \times \boldsymbol{v}}{\|\boldsymbol{r} \times \boldsymbol{v}\|} \quad \hat{\boldsymbol{n}}_{\mathcal{I}}=\hat{\boldsymbol{n}}_{\mathcal{I}} \times \hat{\boldsymbol{t}}_{\mathcal{I}}
$$

A depiction of the two coordinate frames is given in Fig. 1 .

\section{B. Density Transformation Using Change of Variable}

Considering a change of variables from two spaces with equal dimensionality, $\boldsymbol{y}=\boldsymbol{\varphi}(\boldsymbol{x}), \boldsymbol{x}, \boldsymbol{y} \in \mathbb{R}^{m}, \boldsymbol{\varphi}$ being differentiable, the density $p_{y}$ can be derived from $p_{x}$ by integrating over an infinitesimal volume (considering that the integral is invariant under transformation)

$$
p_{y} \mathrm{~d} V_{y}=p_{x} \mathrm{~d} V_{x}
$$

and using integration by substitution, such that ([20] Chap. 5)

$$
p_{y}(\boldsymbol{y})=\frac{p_{x}\left(\boldsymbol{\varphi}^{-1}(\boldsymbol{y})\right)}{|\operatorname{det} \boldsymbol{J}|}
$$

with the Jacobian $\boldsymbol{J} \in \mathbb{R}^{m \times m}$, defined as

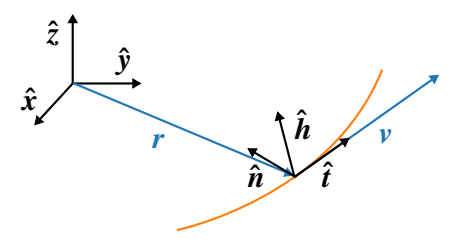

Fig. 1 Satellite frame $\mathcal{T}=\{\hat{t}, \hat{n}, \hat{h}\}$ relative to the inertial frame $\mathcal{I}=\{\hat{x}, \hat{y}, \hat{z}\}$. 


$$
J_{i j}=\frac{\partial \varphi_{i}}{\partial x_{j}}
$$

Some care needs to be taken if the function $\varphi$ is not invertible; i.e., an output $\boldsymbol{y}$ results from multiple different inputs $\boldsymbol{x}_{k}$. The probability then is the sum of all the possible inputs:

$$
p_{\boldsymbol{y}}(\boldsymbol{y})=\sum_{k} \frac{p_{\boldsymbol{x}}\left(\boldsymbol{x}_{k}\right)}{\left|\operatorname{det} \boldsymbol{J}_{k}\right|}
$$

Transformations of densities from a higher- to a lower-dimensional space using the change of variables approach involve integration of joint probabilities to find the marginal probability. Such joint probabilities cannot always be found. Alternatively, the variables can be transformed using the Dirac generalized function, introduced in the next section (Sec. II.C). Transformations from lower- to higher-dimensional spaces are omitted here because they produce densities restricted to a lower-dimensional hypersurface. The interested reader is referred to the literature about geometric measure theory (e.g., [35] Chap. 3).

\section{Density Transformation Using Dirac Generalized Function}

For the purpose of estimating the spatial density or representation of results, it is of interest to map a higher-dimensional distribution into two or even one dimension. Instead of integrating in the target space, the distribution can be directly transformed through integration of the initial space using the Dirac generalized function (e.g. see [34]). Given a continuous transformation $\boldsymbol{y}=\varphi(\boldsymbol{x})$, with $\boldsymbol{\varphi}: \mathbb{R}^{m} \rightarrow$ $\mathbb{R}^{w}$ and $m>w$, the distribution function in $\boldsymbol{y}$ can be found by integrating over $\mathbb{R}^{m}$ as

$$
\begin{aligned}
p_{\boldsymbol{y}} & =\int_{\mathbb{R}^{m}} p_{\boldsymbol{x}} \times \delta[\boldsymbol{\varphi}(\boldsymbol{x})-\boldsymbol{y}] \mathrm{d}^{m} \boldsymbol{x} \\
& =\int_{\mathbb{R}^{m}} p_{\boldsymbol{x}} \times \delta\left[\varphi_{1}(\boldsymbol{x})-y_{1}\right] \cdots \times \delta\left[\varphi_{w}(\boldsymbol{x})-y_{w}\right] \mathrm{d} x_{1} \ldots \mathrm{d} x_{m}
\end{aligned}
$$

where $\delta[\cdot]$ is the Dirac delta function

$$
\delta(x)= \begin{cases}1, & \text { if } x=0 \\ 0, & \text { otherwise }\end{cases}
$$

with the properties

$$
\begin{aligned}
& \int_{-\infty}^{\infty} f(x) \delta[x-a] \mathrm{d} x=f(a) \\
& \delta[g(x)]=\sum_{i}\left|\frac{\partial g}{\partial x}\left(x_{i}\right)\right|^{-1} \delta\left[x-x_{i}\right] \quad g\left(x_{i}\right)=0, \quad \frac{\partial g}{\partial x}\left(x_{i}\right) \neq 0
\end{aligned}
$$

The evaluation requires to find the roots of $\varphi(x)-y=0$. Hence, the method intrinsically deals with noninvertible functions. Each root corresponds to a possible instance of $\boldsymbol{x}_{k}$ that is transformed into $\boldsymbol{y}$.

To promote the understanding of its application, an example is given here, transforming the probability function $p_{v}$, in $\boldsymbol{v}=\left(v_{x} v_{y} v_{z}\right)^{T} \in$ $\mathbb{R}^{3}$ to $p_{a, e}$, in $(a, e) \in \mathbb{R}^{2}$, around the point $\boldsymbol{r}=\left(r_{x} r_{y} r_{z}\right)^{T}$, i.e.,

$$
\begin{aligned}
p_{a, e}= & \int_{\mathbb{R}^{3}} p_{v} \times \delta\left[\varphi_{a}\left(v_{x}, v_{y}, v_{z}\right)-a\right] \\
& \times \delta\left[\varphi_{e}\left(v_{x}, v_{y}, v_{z}\right)-e\right] \mathrm{d} v_{x} \mathrm{~d} v_{y} \mathrm{~d} v_{z}
\end{aligned}
$$

The roots in, say, $v_{x}$ and $v_{y}$, given $\boldsymbol{r}, a, e$, and $v_{z}$, are found using the following relationships ([36] p. 501 and [33] p. 27):

$$
\boldsymbol{r} \cdot \boldsymbol{v}=\frac{\mu}{h} r e \sin f=c
$$

$$
v^{2}=v_{x}^{2}+v_{y}^{2}+v_{z}^{3}=\frac{2 \mu}{r}-\frac{\mu}{a}
$$

with the gravitational parameter $\mu$, arbitrarily introduced constant $c$, and the specific angular momentum $h$, which can be calculated via the semiparameter $p$ as

$$
\begin{aligned}
& p=a\left(1-e^{2}\right) \\
& h=\sqrt{\mu p}
\end{aligned}
$$

Eliminating $v_{x}$ by inserting Eq. (13b) in the squared form of Eq. (13a) allows to find a quadratic expression in, say, $v_{y}$, e.g.,

$$
\left(r_{x}^{2}+r_{y}^{2}\right) v_{y}^{2}+2 r_{y}\left(r_{z} v_{z}-c\right) v_{y}+\left(c-r_{z} v_{z}\right)^{2}+r_{x}^{2}\left(v_{z}^{2}-v^{2}\right)=0
$$

Solving for the two roots $v_{y}^{+}$and $v_{y}^{-}$and inserting them into either of the quadratic Eq. (13b) or Eq. (13a) give the four roots in $v_{x}$ :

$$
\begin{array}{ll}
v_{x}^{++}=v_{x}^{+}\left(v_{y}^{+}\right) & v_{x}^{+-}=v_{x}^{-}\left(v_{y}^{+}\right) \\
v_{x}^{-+}=v_{x}^{+}\left(v_{y}^{-}\right) & v_{x}^{--}=v_{x}^{-}\left(v_{y}^{-}\right)
\end{array}
$$

Special care needs to be taken for cases where $r_{x}$ vanishes, which in the $\mathcal{T}$ frame occurs for circular orbits or around the apsides. Then, Eq. (13a) becomes decoupled from $v_{x}$, and $v_{y}$ can be directly inferred. Finally, the distribution $p_{a, e}$ is found as

$$
p_{a, e}=\int_{\mathbb{R}} \sum_{l \in \pm} \sum_{\kappa \in \pm} \frac{1}{\left|\operatorname{det} \boldsymbol{J}\left(v_{x}^{l \kappa}, v_{y}^{l}\right)\right|} p_{v}\left(v_{x}^{i \kappa}, v_{y}^{l}, v_{z}\right) \mathrm{d} v_{z}
$$

where

$$
\boldsymbol{J}\left(v_{x}^{l \kappa}, v_{y}^{l}\right)=\left.\left(\begin{array}{ll}
\frac{\partial a}{\partial v_{x}} & \frac{\partial a}{\partial v_{y}} \\
\frac{\partial e}{\partial v_{x}} & \frac{\partial e}{\partial v_{y}}
\end{array}\right)\right|_{\substack{v_{x}=v_{x}^{I K} \\
v_{y}=v_{y}^{l}}}
$$

and the derivatives of $a$ and $e$ with respect to $v_{x}$ and $v_{y}$ can be found in literature (e.g., [37]). The same result could be achieved by integration of $p_{a, e, i}$, obtained from $p_{v}$ using Eq. (6), over $i$. However, the Dirac generalized method is more generally applicable, even if the joint distribution in $p_{a, e, i}$ was not available.

\section{Density Transformation from Cartesian Coordinates to Keplerian Elements}

If the partial derivatives of a transformation between two spaces of the same dimension are known, the transformation of the density function can be obtained through application of Eq. (6). A compilation of partial derivatives of transformations between $\bar{C}$ artesian coordinates, spherical coordinates, Keplerian elements, and equinoctial elements is given in [37] for the spaces defined relative to the $\mathcal{I}$ frame. It is convenient to work in the $\mathcal{T}$ frame if impulses are to be added, as most maneuvers are executed relative to $\boldsymbol{v}$. Transformations into Keplerian elements are discussed here due to the widespread use and physical interpretability of the elements.

The Jacobian of the Keplerian elements with respect to $s$, evaluated

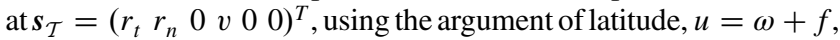
and abbreviating $s_{\gamma}=\sin \gamma$ and $c_{\gamma}=\cos \gamma$, is [38]

$$
\boldsymbol{J}_{\alpha s_{\mathcal{T}}}=\left.\frac{\partial \boldsymbol{\alpha}}{\partial \boldsymbol{s}}\right|_{\mathcal{T}}=\left(\left.\left.\frac{\partial \boldsymbol{\alpha}}{\partial \boldsymbol{r}}\right|_{\mathcal{T}} \frac{\partial \boldsymbol{\alpha}}{\partial \boldsymbol{v}}\right|_{\mathcal{T}}\right)
$$




$$
=\left(\begin{array}{cccccc}
\frac{2 a^{2}}{r^{3}} r_{t} & \frac{2 a^{2}}{r^{3}} r_{n} & 0 & \frac{2 a^{2} v}{\mu} & 0 & 0 \\
G_{21} r_{t}+\frac{r s_{f}}{a h} v & G_{21} r_{n} & 0 & \frac{2\left(e+c_{f}\right)}{v} & -\frac{r s_{f}}{a v} & 0 \\
0 & 0 & \frac{s_{u}+e s_{\omega}}{p} & 0 & 0 & \frac{r c_{u}}{h} \\
0 & 0 & -\frac{c_{u}+e c_{\omega}}{p s_{i}} & 0 & 0 & \frac{r s_{u}}{h s_{i}} \\
G_{51} r_{t}-\frac{r\left(c_{f}+e\right)}{\mathrm{hep}} v & G_{51} r_{n} & \frac{\left(c_{u}+e c_{\omega}\right) c_{i}}{p s_{i}} & \frac{2 s_{f}}{e v} & \frac{2}{v}+\frac{r c_{f}}{\mathrm{eav}} & -\frac{r s_{u}}{h \tan i} \\
G_{61} r_{t}+G_{62} v & G_{61} r_{n} & 0 & -\frac{2 s_{f}}{e v} & -\frac{2}{v}-\frac{r c_{f}}{\mathrm{eav}} & 0
\end{array}\right)
$$

$$
=\left(J_{\boldsymbol{\alpha} r_{T}} \boldsymbol{J}_{\boldsymbol{\alpha} v_{T}}\right)
$$

where $\boldsymbol{J}_{\boldsymbol{\alpha} r_{\mathcal{T}}}, \boldsymbol{J}_{\boldsymbol{\alpha} v_{\mathcal{T}}} \in \mathbb{R}^{6 \times 3}$ relate $\boldsymbol{r}_{\mathcal{T}}$ and $\boldsymbol{v}_{\mathcal{T}}$ to $\boldsymbol{\alpha}$, respectively, and

$$
\begin{aligned}
G_{21} & =\frac{1}{\mu a e}\left(\frac{h^{2} a}{r^{3}}-v^{2}\right) \\
G_{51} & =-\frac{r \sin f}{h^{2} e}\left(\frac{h^{2}\left(p+e^{2} r\right)}{p r^{3}}-\frac{(p+r) v^{2}}{r^{2}}\right) \\
G_{61} & =\frac{r \sin f}{h^{2} e} \frac{\mu}{a r^{3}}((r-a)(p+r)-r a) \\
G_{62} & =\frac{r}{h^{2} e}\left(\frac{h\left(c_{f}+e\right)}{p}+\frac{h e}{r}\right)
\end{aligned}
$$

Note that $\boldsymbol{J}_{\boldsymbol{\alpha} s_{\mathcal{T}}}$ is singular for $e=0$ and/or $i=0 / 180 \mathrm{deg}$, for which some of the Keplerian elements are ill-defined. For the case of fragmentations, only submatrices of $\boldsymbol{J}_{\boldsymbol{\alpha} s_{\mathcal{T}}}$ are required, some of which are nonsingular for any $e$ or $i$, such as the Jacobian relating the velocity components to $(a, e, i)$. Instead, this transformation is singular at the perigee and apogee, i.e., at $f=0 / 180 \mathrm{deg}$, and $90 \mathrm{deg}$ away from the crossing of the equatorial plane, i.e., at $u= \pm 90 \mathrm{deg}$, as it reduces the rank of the submatrix to below its dimension. Still, the transformation of a distribution of fragments stemming from a parent object residing on one of those singularities can be performed in Keplerian elements, as the fragments themselves are ejected out of the singularity. Furthermore, even for nonsingular elements, such as equinoctial elements [39], the submatrices have a rank that is lower than the dimension in the aforementioned singularities. Hence, opposite to what their name suggests, nonsingular elements do not solve the problem of singularity during transformation.

The density in Keplerian elements, $p_{\alpha}$, can now be found from the density given in Cartesian coordinates, $p_{s}$, by plugging $J_{\alpha s_{\mathcal{T}}}$ into Eq. (ㅁ) :

$$
p_{\alpha}=\frac{p_{s}\left(\boldsymbol{\varphi}_{s_{\mathcal{T}} \boldsymbol{\alpha}}(\boldsymbol{\alpha})\right)}{\left|\operatorname{det} \boldsymbol{J}_{\boldsymbol{\alpha s}_{\mathcal{T}}}\right|}
$$

Equation (21) is valid for any Cartesian frame that is obtained by rotation from $\overline{\mathcal{T}}$, as the volume is invariant under rotation. Thus, no specific frame is mandated for the transformation $\varphi_{s \alpha}$ if it matches the frame in which $p_{s}$ is defined. To increase readability, the subscript $\mathcal{T}$ is dropped from the equations for the remainder of this work.

Equation (21) transforms a distribution from Cartesian coordinates into oscillating Keplerian elements, $\boldsymbol{\alpha}$. The propagation, instead, is preferably performed in mean Keplerian elements, $\overline{\boldsymbol{\alpha}}$. Oscillating elements can be obtained from mean orbital elements through

$$
\alpha=\bar{\alpha}+\Delta \alpha(\bar{\alpha})
$$

where $\Delta \boldsymbol{\alpha}$ contains the short-period variations, which depend on the considered force model. For example, in his artificial satellite theory, Brouwer finds $\Delta \boldsymbol{\alpha}$ analytically considering the perturbations from the second-order zonal harmonic, $J_{2}$ [40]. Usually no explicit transformations from oscillating to mean elements are available, making iterations necessary. Given Eq. (22), a distribution given in $\alpha, p_{\alpha}$, can be transformed into a distribution given in $\overline{\boldsymbol{\alpha}}, p_{\overline{\boldsymbol{\alpha}}}$, using Eq. (ㅁ) as

$$
p_{\overline{\boldsymbol{\alpha}}}=p_{\alpha}(\overline{\boldsymbol{\alpha}}+\Delta \boldsymbol{\alpha})\left|\operatorname{det} \boldsymbol{J}_{\boldsymbol{\alpha} \overline{\boldsymbol{\alpha}}}\right|
$$

with

$$
\boldsymbol{J}_{\boldsymbol{\alpha} \overline{\boldsymbol{\alpha}}}=\left(\frac{\partial \alpha_{i}}{\partial \bar{\alpha}_{j}}\right)_{i, j=1, \ldots, 6}
$$

Plugging in Eq. (22) shows that

$$
\frac{\partial \alpha_{i}}{\partial \bar{\alpha}_{j}}=\delta_{i j}+\frac{\partial \Delta \alpha_{i}}{\partial \bar{\alpha}_{j}} \quad \forall i, j=1, \ldots, 6
$$

where $\delta_{i j}$ is the Kronecker delta. Noticing that $\left(\partial \Delta \alpha_{i} / \partial \bar{\alpha}_{j}\right) \ll 1$, e.g., of the order of $J_{2}$ in Brouwer's theory, the Jacobian relating the mean and oscillating state approximates the $6 \times 6$ identity matrix [41]. Hence, Eq. (23) can be approximated as

$$
p_{\bar{\alpha}} \approx p_{\alpha}(\overline{\boldsymbol{\alpha}}+\Delta \boldsymbol{\alpha})
$$

The fragments obtained from the breakup model discussed in Sec. III span large domains in $\boldsymbol{\alpha}$. Hence, it is justified to ignore the relatively small displacements of $\Delta \boldsymbol{\alpha}$ in the transformation to mean elements.

It is important to mention that evaluating $\boldsymbol{J}_{\boldsymbol{\alpha} s}$ in SI units leads to an ill-conditioned matrix. Calculating the determinant of such a matrix might induce numerical errors. Therefore, it is advised to evaluate $\boldsymbol{J}_{\boldsymbol{\alpha} s}$ using adimensional variables, e.g.,

$$
\hat{a}=\hat{\mu}=1 \rightarrow \hat{\boldsymbol{r}}=\frac{\boldsymbol{r}}{a}, \quad \hat{\boldsymbol{v}}=\frac{\boldsymbol{v}}{\sqrt{\mu / a}}, \text { etc }
$$

The resulting adimensional Jacobian then needs to be renormalized again.

\section{E. Density Transformation from Keplerian Elements to Cartesian Coordinates}

The fragment cloud potentially poses a risk to targets orbiting nearby. The estimation of the number of impacts (and thus the collision probability) is more conveniently performed in Cartesian coordinates 
(see Sec. IV). Hence, given a distribution in Keplerian elements, the inverse transformation is required. The inverse density transformation can be found by deriving the Jacobian of $\boldsymbol{s}$ with respect to $\boldsymbol{\alpha}$, or more straightforwardly, it can be derived by inverting Eq. (21)

$$
p_{s}=p_{\alpha}\left(\boldsymbol{\varphi}_{\alpha s}(\boldsymbol{s})\right)\left|\operatorname{det} \boldsymbol{J}_{\alpha s}\right|
$$

where

$$
\varphi_{\alpha s}=\varphi_{s \alpha}^{-1}
$$

relates a Cartesian state to its Keplerian elements (e.g., [33] Chap. 2.7)

\section{Reformulation of the NASA Standard Breakup Model}

\section{A. Probabilistic Description}

The NASA SBM [24] can be used to sample deterministic fragments following an explosion or collision event in orbit. It is a semi-empirical model based on evidence compiled from historical orbital data and ground-based impact tests. The samples are described in characteristic length $L$, area-to-mass ratio $A / m$, and the ejection velocity $\Delta v$ imparted in random direct relative to the parent orbital velocity. Here the NASA SBM is reformulated as a probability distribution function. The number of objects with $L$ larger than a lower bound $L_{0}$ produced in the fragmentation event is

$$
N_{L}\left(L_{0}\right)=k\left(\frac{L_{0}[\mathrm{~m}]}{1[\mathrm{~m}]}\right)^{-\beta} \quad k, \beta>0
$$

where $k$ and $\beta$ are unitless parameters dependent on the type of fragmentation and the physical characteristics of the involved objects. All the parameters introduced in this section are given in Appendix B. In the following, the normalization in the units, e.g.,

$$
\frac{L_{0}[\mathrm{~m}]}{1[\mathrm{~m}]}
$$

is omitted. Each variable is, if not stated otherwise, defined in SI units. Equation (30) can be converted into a probability density function as

$$
p_{L}=\frac{k \beta}{N} L^{-(\beta+1)} \quad \forall L_{0} \leq L \leq L_{1}
$$

where $L_{1}$ is the upper boundary on the characteristic length, and

$$
N=N_{L}\left(L_{0}\right)-N_{L}\left(L_{1}\right)
$$

is the total number of fragments within this range. Combining Eqs. (32) and $(\underline{33})$ as

$$
p_{L}=\beta \frac{L^{-(\beta+1)}}{L_{0}^{-\beta}-L_{1}^{-\beta}} \quad \forall L_{0} \leq L \leq L_{1}
$$

reveals that $p_{L}$ is independent of the number of fragments involved and thus solely dependent on the power factor $\beta$. Still, all the probability distribution functions derived herein can be multiplied by $N$ to obtain the fragment phase space densities.

The conditional probabilities introducing the dependence on $L$, $A / m$, and $\Delta v$ are given as a function of the logarithm to base 10 of the characteristic length, $\lambda=\log _{10}(L)$; the logarithm to base 10 of the area-to-mass ratio, $\chi=\log _{10}(\mathrm{~A} / \mathrm{m})$; and the logarithm to base 10 of the absolute impulse, $v=\log _{10}(\Delta v)$. Transforming Eq. (32) into a function dependent on $\lambda$ using Eq. (6) yields

$$
p_{\lambda}=\log (10) 10^{\lambda} p_{L}\left(10^{\lambda}\right) \quad \forall \lambda_{0}=\log _{10}\left(L_{0}\right) \leq \lambda \leq \lambda_{1}=\log _{10}\left(L_{1}\right)
$$

$$
=\log (10) \beta \frac{10^{-\beta \lambda}}{10^{-\beta \lambda_{0}-10^{-\beta \lambda_{1}}}}
$$

To sample $\lambda$ from Eq. ( $\underline{35 b})$, the cumulative distribution function

$$
P_{\lambda}=\int_{\lambda_{0}}^{\lambda} p_{\lambda}\left(\lambda^{\prime}\right) \mathrm{d} \lambda^{\prime}=\frac{10^{-\beta_{\lambda 0}}-10^{-\beta \lambda}}{10^{-\beta_{\lambda 0}}-10^{-\beta \lambda_{1}}}
$$

is inverted

$$
\lambda=-\frac{1}{\beta} \log _{10}\left[10^{-\beta \lambda_{0}}-P_{\lambda}\left(10^{-\beta \lambda_{0}}-10^{-\beta \lambda_{1}}\right)\right]
$$

Equation (37) enables the sampling of $\lambda$ by sampling $P_{\lambda}$ uniformly $\in[0,1]$. The conditional probabilities in $\chi$ and $\nu$, dependent on $\lambda$ and $\chi$, respectively, are as sum of normal distributions, $\mathcal{N}$, as [24]

$$
\begin{aligned}
& p_{\chi \mid \lambda}=\sum_{i} \alpha_{i}(\lambda) \mathcal{N}\left(\mu_{\chi}^{(i)}(\lambda), \sigma_{\chi}^{(i)}(\lambda)\right) \quad \sum_{i} \alpha_{i}(\lambda)=1 \\
& p_{v \mid \chi}=\mathcal{N}\left(\mu_{v}(\chi), \sigma_{v}(\chi)\right)
\end{aligned}
$$

where, again, the functions $\alpha_{i}, \mu_{\chi}^{(i)}, \sigma_{\chi}^{(i)}, \mu_{v}, \sigma_{v}$ are dependent on the type of fragmentation, i.e., explosion or fragmentation, and the type of the involved objects, i.e., payload or rocket body. Most relevant mathematical libraries provide functions to sample normal distributions. The joint probability in $\lambda, \chi$, and $v$ is

$$
p_{\lambda, \chi, v}=p_{v \mid \chi} p_{\chi \mid \lambda} p_{\lambda}
$$

Marginal distributions can be obtained by numerical integration, e.g.,

$$
\begin{aligned}
p_{\chi, v} & =p_{v \mid \chi} \int_{\lambda_{0}}^{\lambda_{1}} p_{\chi \mid \lambda} p_{\lambda} \mathrm{d} \lambda \\
p_{v} & =\int_{\mathbb{R}} p_{\chi, v} \mathrm{~d} \chi
\end{aligned}
$$

Finally, the distributions in $L, A / m$, and $\Delta v$ and their marginals can be found through application of Eq. (6):

$p_{L, \frac{A}{m}, \Delta v}=\frac{p_{\lambda, \chi, v}\left(\log _{10}(L), \log _{10}\left(\frac{A}{m}\right), \log _{10}(\Delta v)\right)}{\log ^{3}(10) L \frac{A}{m} \Delta v} L_{0} \leq L \leq L_{1}$

$$
\begin{aligned}
p_{\frac{A}{m}, \Delta v}= & \frac{p_{\chi, v}\left(\log _{10}\left(\frac{A}{m}\right), \log _{10}(\Delta v)\right)}{\log ^{2}(10) \frac{A}{m} \Delta v} \\
p_{\Delta v}= & \frac{p_{v}\left(\log _{10} \Delta v\right)}{\log (10) \Delta v}
\end{aligned}
$$

The cross-sectional area $A$ and the mass $m$ follow directly from the previously introduced random variables as

$$
\begin{aligned}
& A=b L^{\gamma} \\
& m=\frac{A}{A / m}
\end{aligned}
$$

where $b$ and $\gamma$ are parameters dependent on $L$, given in Appendix B. To introduce a directional component, the impulse distribution $p_{\Delta v}$ needs to be multiplied by a directional distribution $p_{\phi, \varphi \mid \Delta v}$ with the spherical angles 


$$
\phi \in[0,2 \pi) \quad \varphi \in\left[-\frac{\pi}{2}, \frac{\pi}{2}\right]
$$

defined relative to the $\mathcal{T}$ frame. To find $p_{\phi, \varphi \mid \Delta v}$, isotropic direction is assumed. Each infinitely small area $\mathrm{d} S$ on the sphere defined by the radius $\Delta v$ is equally likely; hence

$$
p_{S}=\frac{1}{4 \pi(\Delta v)^{2}}
$$
in

Using $\mathrm{d} S=(\Delta v)^{2} \cos \varphi \mathrm{d} \phi \mathrm{d} \varphi$ and application of Eq. (므), resulting

$$
p_{\phi, \varphi \mid \Delta v} \mathrm{~d} \phi \mathrm{d} \varphi=p_{S} \mathrm{~d} S=\frac{\cos \varphi}{4 \pi} \mathrm{d} \phi \mathrm{d} \varphi
$$

shows that

$$
p_{\phi, \varphi \mid \Delta v}=p_{\phi, \varphi}=\frac{\cos \varphi}{4 \pi}
$$

The marginals

$$
\begin{aligned}
& p_{\phi}=\int_{-\frac{\pi}{2}}^{\frac{\pi}{2}} p_{\phi, \varphi} \mathrm{d} \varphi=\frac{1}{2 \pi} \\
& p_{\varphi}=\int_{0}^{2 \pi} p_{\phi, \varphi} \mathrm{d} \phi=\frac{\cos \varphi}{2}
\end{aligned}
$$

show that only $\phi$ is uniformly distributed over $\phi \in[0,2 \pi)$, but $\varphi$ is not uniformly distributed. To sample $\varphi$, the cumulative distribution function $P_{\varphi}$, given as

$$
P_{\varphi}=\int_{-\frac{\pi}{2}}^{\varphi} p_{\varphi}\left(\varphi^{\prime}\right) \mathrm{d} \varphi^{\prime}=\frac{1}{2}(\sin \varphi+1)
$$

needs to be solved for $\varphi$, such that

$$
\sin \varphi=2 P_{\varphi}-1
$$

Hence, the distribution in spherical velocity coordinates is

$$
p_{\Delta v, \phi, \varphi}=\frac{\cos \varphi}{4 \pi} p_{\Delta v}
$$

Transformation $p_{\Delta v, \phi, \varphi}$ into Cartesian velocity coordinates in the $\mathcal{T}$ frame, given as

$$
\begin{aligned}
& \Delta v_{t}=\Delta v \cos \varphi \cos \phi \\
& \Delta v_{n}=\Delta v \cos \varphi \sin \phi \\
& \Delta v_{h}=\Delta v \sin \varphi
\end{aligned}
$$

and, using Eq. (6), results in the directional distribution

$$
p_{\Delta v}=\frac{p_{\Delta v, \phi, \varphi}}{\left(\Delta v^{2}\right) \cos \varphi}=\frac{p_{\Delta v}}{4 \pi(\Delta v)^{2}}
$$

Finally, a simple translation results in the desired density function

$$
p_{v}\left(v^{+}\right)=p_{\Delta v}\left(v^{+}-v^{-}\right)
$$

where $\boldsymbol{v}^{-}$and $\boldsymbol{v}^{+}$are the orbital velocities immediately before and after the impulse. As the fragments are ejected equally likely in any direction, the frame can be chosen freely.

\section{B. Expectation Values}

The NASA SBM does not inherently conserve physical quantities such as mass and kinetic energy [42]. Given a fully probabilistic description of the breakup model permits to check the conservation, or enforce it, via the calculation of expectation values. In general, the expectation value $\mathbb{E}$ of a function $g(\boldsymbol{x})$ of the $m$-dimensional continuous random variable $\boldsymbol{x} \in \mathbb{R}^{m}$ and its joint probability density function $p_{x}$ is

$$
\mathbb{E}(g(\boldsymbol{x}))=\int_{\mathbb{R}^{m}} g(\boldsymbol{x}) p_{\boldsymbol{x}} \mathrm{d}^{m} \boldsymbol{x}
$$

The integration can be performed in any frame. As the distributions are mostly defined with regards to $(\lambda, \chi, v)$, the expectation values are most easily integrated in this frame too. The mass $m$ and kinetic energy of the impulse, $\Delta \epsilon$, are calculated as

$$
\begin{aligned}
m & =\frac{A}{A / m}=b 10^{\lambda \gamma-\chi} \\
\Delta \epsilon & =\frac{1}{2} m \Delta v^{2}=\frac{b}{2} 10^{\lambda \gamma-\chi+2 v}
\end{aligned}
$$

Hence, their expectation values are

$$
\begin{aligned}
\mathbb{E}(m) & =\int_{\mathbb{R}} \int_{\lambda_{0}}^{\lambda_{1}} b 10^{\lambda \gamma-\chi} p_{\chi \mid \lambda} p_{\lambda} \mathrm{d} \lambda \mathrm{d} \chi \\
\mathbb{E}(\Delta \epsilon) & =\frac{1}{2} \int_{\mathbb{R}} \int_{\mathbb{R}} \int_{\lambda_{0}}^{\lambda_{1}} b 10^{\lambda \gamma-\chi+2 v} p_{v \mid \chi} p_{\chi \mid \lambda} p_{\lambda} \mathrm{d} \lambda \mathrm{d} \chi \mathrm{d} v
\end{aligned}
$$

The expectation values for the different fragmentation scenario are given in Table 1 for different bins in $L$, alongside the respective shares, $P_{L}$, which is the integral over the given bins assuming $L_{0}=1 \mathrm{~mm}$ and $L_{1}=1 \mathrm{~m}$. Note that these values are independent of the total number of fragments, $N$, given from Eq. (33). Instead, they can be used to set $N$ to enforce conservation in either mass or kinetic energy. Conservation of mass means that the sum of the mass of all the fragments equals the fragmenting mass $M_{i}$, i.e.,

$$
M_{i}=N_{m} \mathbb{E}(m)
$$

where $N_{m}$ is the total number of fragments respecting mass conservation. The fragmenting mass is not simply the mass of the parent object, as 2-8 fragments with $L>1 \mathrm{~m}$, not following the power law in Eq. (30), comprise the bulk of the mass [43]. For example, for a payload collision, the mean mass for $1 \mathrm{~mm}<L<$ $1 \mathrm{~m}$ is $\mathbb{E}(m)=4.13 \times 10^{-4} \mathrm{~kg}$. If the mass is set at $M_{i}=100 \mathrm{~kg}$ it follows from Eq. (57) that $N_{m}=2.4 \times 10^{5}$, given that the combined mass in fragments smaller than $L<1 \mathrm{~mm}$ is negligible. The number of fragments required to assure conservation of kinetic energy is harder to estimate as losses due to heat and rotational velocities are not known in this simple model [42]. Luckily, the cloud evolution, from initial distribution, through propagation to estimation of the number of impacts can be performed based on the distribution of a single object only. Once an accurate estimate of $N$ is available, the results can be updated through multiplication with $N$.

\section{Covariance in Velocity}

For methods based on covariance propagation (e.g., see [30]), the initial mean and covariance of the distribution are required. Here, the mean and variance in $\Delta v_{h}$ are derived from the NASA SBM. As isotropic directionality is assumed, the presented values are also applicable in any other direction. To derive any moment in $\Delta v_{h}$, its probability distribution function is required. As becomes apparent later, it is beneficial to transform the distribution in Eq. (2) into cylindrical coordinates 
Table 1 Expectation values for the mass and kinetic energy, and variance for the velocity impulse of the fragments according to the NASA SBM

\begin{tabular}{|c|c|c|c|c|c|c|}
\hline Case & & $L, \mathrm{~m}$ & $P_{L}$ & $\mathbb{E}(m), \mathrm{kg}$ & $\mathbb{E}(\Delta \epsilon), \mathrm{J}$ & $\sigma_{\Delta v_{h}}^{2}, \mathrm{~m}^{2} / \mathrm{s}^{2}$ \\
\hline \multirow{5}{*}{ Collision } & & $10^{-2}-10^{-1}$ & $1.91 \times 10^{-2}$ & $3.67 \times 10^{-3}$ & $2.40 \times 10^{2}$ & $1.21 \times 10^{6}$ \\
\hline & & $10^{-3}-10^{-0}$ & 1 & $4.13 \times 10^{-4}$ & $1.22 \times 10^{1}$ & $7.31 \times 10^{5}$ \\
\hline & Rocket body & $10^{-3}-10^{-2}$ & $9.81 \times 10^{-1}$ & $8.46 \times 10^{-6}$ & $3.75 \times 10^{0}$ & $7.23 \times 10^{5}$ \\
\hline & & $10^{-2}-10^{-1}$ & $1.91 \times 10^{-2}$ & $3.47 \times 10^{-3}$ & $2.51 \times 10^{2}$ & $1.22 \times 10^{6}$ \\
\hline & & $10^{-3}-10^{-0}$ & 1 & $1.78 \times 10^{-4}$ & $1.80 \times 10^{1}$ & $7.32 \times 10^{5}$ \\
\hline \multirow[t]{5}{*}{ Explosion } & Payload & $10^{-3}-10^{-2}$ & $9.75 \times 10^{-1}$ & $9.24 \times 10^{-6}$ & $7.81 \times 10^{-2}$ & $7.18 \times 10^{3}$ \\
\hline & & $10^{-2}-10^{-1}$ & $2.45 \times 10^{-2}$ & $4.16 \times 10^{-3}$ & $1.70 \times 10^{1}$ & $6.83 \times 10^{3}$ \\
\hline & & $10^{-1}-10^{-0}$ & $6.15 \times 10^{-4}$ & $9.92 \times 10^{-1}$ & $2.86 \times 10^{3}$ & $3.95 \times 10^{3}$ \\
\hline & & $10^{-3}-10^{-0}$ & 1 & $7.21 \times 10^{-4}$ & $2.25 \times 10^{0}$ & $7.15 \times 10^{3}$ \\
\hline & Rocket body & $10^{-3}-10^{-2}$ & $9.75 \times 10^{-1}$ & $9.24 \times 10^{-6}$ & $7.81 \times 10^{-2}$ & $7.18 \times 10^{3}$ \\
\hline
\end{tabular}

$$
\begin{aligned}
\Delta v_{\perp} & =\sqrt{(\Delta v)^{2}-\left(\Delta v_{h}\right)^{2}} \\
\phi & =\sin ^{-1}\left(\frac{\Delta v_{h}}{\Delta v}\right) \\
\Delta v_{h} & =\Delta v_{h}
\end{aligned}
$$

yielding under application of Eq. (ㅁ), the distribution

$$
p_{\Delta v_{\perp}, \phi, \Delta v_{h}}=\Delta v_{\perp} p_{\Delta v}=\frac{\Delta v_{\perp}}{4 \pi(\Delta v)^{2}} p_{\Delta v}
$$

Noticing that $p_{\Delta v_{\perp}, \phi, \Delta v_{h}}$ is not dependent on $\phi$, it can be readily reduced as

$$
p_{\Delta v_{\perp}, \Delta v_{h}}=\int_{0}^{2 \pi} p_{\Delta v_{\perp}, \phi, \Delta v_{h}} \mathrm{~d} \phi=\frac{\Delta v_{\perp}}{2(\Delta v)^{2}} p_{\Delta v}
$$

Thanks to the reduction, the distribution in $\Delta v_{h}$ requires integration in only one, rather than two, variables

$$
p_{\Delta v_{h}}=\int_{0}^{\infty} p_{\Delta v_{\perp}, \Delta v_{h}} \mathrm{~d} \Delta v_{\perp}
$$

From Eq. (58a) it follows that $\Delta v_{\perp} \mathrm{d} \Delta v_{\perp}=\Delta v \mathrm{~d} \Delta v$. Putting together the pieces allows to find

$$
p_{\Delta v_{h}}=\int_{\left|\Delta v_{h}\right|}^{\infty} \frac{p_{\Delta v}}{2 \Delta v} \mathrm{~d} \Delta v
$$

The expectation value of $\Delta v_{h}$ is always zero as $p_{\Delta_{h}}\left(\Delta_{h}\right)=$ $p_{\Delta_{h}}\left(-\Delta_{h}\right)$ is symmetrical, i.e.,

$$
\mathbb{E}\left(\Delta v_{h}\right)=\int_{-\infty}^{\infty} \Delta v_{h} p_{\Delta v_{h}} \mathrm{~d} \Delta v_{h}=0
$$

The variance $\sigma_{\Delta v_{h}}^{2}$ can be found as

$$
\begin{aligned}
\sigma_{\Delta v_{h}}^{2}=\operatorname{Var}\left(\Delta v_{h}\right) & =\int_{-\infty}^{\infty}\left(\Delta v_{h}-\mathbb{E}\left(\Delta v_{h}\right)\right)^{2} p_{\Delta v_{h}} \mathrm{~d} \Delta v_{h} \\
& =2 \int_{0}^{\infty}\left(\Delta v_{h}\right)^{2} p_{\Delta v_{h}} \mathrm{~d} \Delta v_{h}
\end{aligned}
$$

where the symmetry is exploited again. Finally, the mean $\boldsymbol{\mu}$ and covariance matrix $\boldsymbol{\Sigma}$ of the NASA SBM velocity distribution are given as

$$
\boldsymbol{\mu}_{\Delta v}=\mathbf{0} \quad \boldsymbol{\Sigma}_{\Delta v}=\operatorname{diag}\left(\sigma_{\Delta v_{t}}^{2} \sigma_{\Delta v_{n}}^{2} \sigma_{\Delta v_{h}}^{2}\right)
$$

where $\sigma_{\Delta v_{t}}^{2}=\sigma_{\Delta v_{n}}^{2}=\sigma_{\Delta v_{h}}^{2}$. The corresponding values for the different cases and various characteristic length regimes (again valid for any $N$ ) are listed in Table 1 . Note that a normal distribution with the given covariance matrix does not accurately describe $p_{\Delta v}$, as can be seen in Fig. 2 for the case of a payload collision. It underestimates both the peak and the tail end of the distribution, while overestimating the domain in between. Hence, application of the covariance needs to be treated with caution.

\section{Spatial Density and Number of Impacts}

The previous two sections discuss how to obtain an initial distribution and transform it into Keplerian elements. Here, the focus is changed to obtain the spatial density and the number of impacts from any distribution in Keplerian elements. The step in between, the density propagation (e.g., see [11]), is not addressed here other than considering randomization in the node and line of apsides due to an oblate central body.

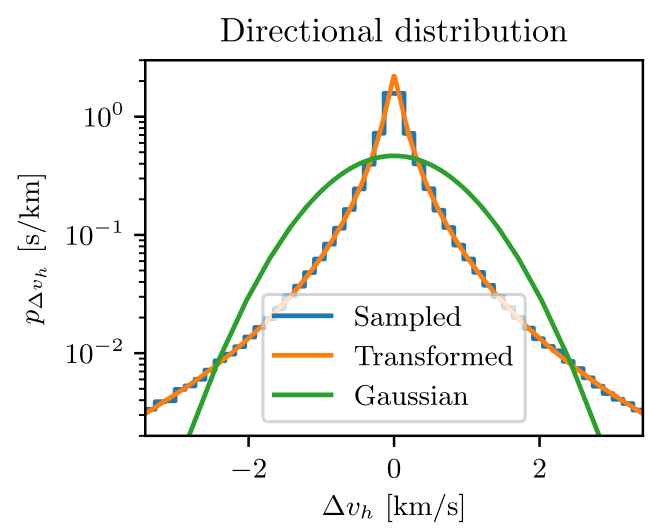

Fig. 2 Transformed and sampled distribution in $\Delta v_{h}$ according to the NASA SBM for a payload collision and fragments sized $1 \mathrm{~mm}<L<1 \mathrm{~m}$, and its comparison with the Gaussian approximation with zero mean and variance, $\sigma_{\Delta v_{h}}^{2}$. 


\section{A. Randomization}

As observed by McKnight and Lorenzen, the evolution of a cloud of fragments in low Earth orbit (LEO) can be considered in phases [7]. Thus, for long-term propagation, only the distribution in $a$ and $e$ is of importance. The randomization in the angles $\Omega, \omega$, and $M$ is used to derive the full phase space density, and spatial density. The application of the assumptions directly in Keplerian elements is straightforward. Each of the randomizations can be applied independently of the other, and thus the extensions are valid for distributions given in any combination of $\alpha$.

Start with a distribution $n_{a, e}$, given as a function of $a$ and $e$. The inclination $i$ of the fragments can be approximated as the parent inclination $i_{0}$. In LEO, this assumption is reasonable because changes in $i$ require large $\Delta v_{h}$ and the perturbing forces on $i$ are small. Thus, the distribution can be extended to

$$
n_{a, e, i}=\delta\left(i-i_{0}\right) n_{a, e}
$$

where $\delta$ is, again, the Dirac generalized function [see Eq. (10)]. Randomization of $\Omega$ due to nodal precession, $\omega$ due to apsidal precession, and $M$ due to differences in energy results in a uniform distribution over $[0,2 \pi)$ each as

$$
\begin{gathered}
n_{a, e, i, \Omega}=\frac{1}{2 \pi} n_{a, e, i} \quad \forall \Omega \in[0,2 \pi) \\
\left.n_{a, e, i, \Omega, \omega}=\frac{1}{2 \pi} n_{a, e, i, \Omega} \quad \forall \omega \in[0,2 \pi]\right) \\
\left.n_{a, e, i, \Omega, \omega, M}=\frac{1}{2 \pi} n_{a, e, i, \Omega, \omega} \quad \forall M \in[0,2 \pi]\right)
\end{gathered}
$$

Thus, the distribution in the full Keplerian element set $\boldsymbol{\alpha}$, using the method of change of variables ([20] Chap. 5), is

$$
\begin{aligned}
n_{\alpha}=n_{a, e, i, \Omega, \omega, f} & =\frac{\mathrm{d} M}{\mathrm{~d} f} n_{a, e, i, \Omega, \omega, M} \\
& \left.=\frac{\left(1-e^{2}\right)^{3 / 2}}{(1+e \cos f)^{2}} n_{a, e, i, \Omega, \omega, M} \forall f \in[0,2 \pi]\right)
\end{aligned}
$$

where the derivative of $M$ with respect to $f$ is given in literature (e.g., [36] Chap. 5.3). Through application of Eq. (28), a distribution in the full Keplerian element set is transformed into Cartesian coordinates. Hence, the phase space density in Cartesian coordinates, $n_{s}$, can be found from a subset of $\alpha$, given the dependence on $a$ and $e$ and assumptions on randomization. The knowledge of the full phase space density enables the derivation of products, such as the spatial density and the impact rate of a fragment cloud on space missions.

\section{B. Spatial Density}

Given the phase space density in Cartesian coordinates, $n_{s}=n_{r, v}$, the spatial density is obtained by integration over the velocities:

$$
n_{r}=\int_{\mathbb{R}^{3}} n_{r, v} \mathrm{~d}^{3} \boldsymbol{v}
$$

It is worth noting that a fixed inclination constraint reduces the possible incident velocities into an ascending and descending crossing plane. Hence, the volume integral in Eq. (69) can be reduced to an area integral by rotating into the satellite frame $\mathcal{T}$ where the probability for nonzero out-of-plane velocities $v_{h}$ is zero. Thus,
Instead, the spatial density can be directly obtained from integration in Keplerian elements using the Dirac generalized function (see Sec. II.C)

$$
n_{\boldsymbol{r}}\left(\boldsymbol{r}^{*}\right)=\int_{\mathbb{R}^{6}} n_{\alpha} \delta\left(\boldsymbol{r}(\boldsymbol{\alpha})-\boldsymbol{r}^{*}\right) \mathrm{d}^{6} \boldsymbol{\alpha}
$$

where $\boldsymbol{r}^{*}$ is the position of interest. To find the roots of $\boldsymbol{r}(\boldsymbol{\alpha})-\boldsymbol{r}^{*}$, it is important to find a three-dimensional subset of $\boldsymbol{\alpha}$ (the integrands) that together with $\boldsymbol{r}^{*}$ permit to find the full $\boldsymbol{\alpha}$. Not all combinations of the elements are possible. It needs to contain one of the elements defining the orientation of the orbital plane, i.e., $i$ or $\Omega$. And it requires any two of the other four elements, excluding the combination of $\omega$ and $f$, because this combination does not permit to calculate the angular momentum or mechanical energy of the orbit. Here, the subset of $a, e$, and $i$ is chosen because the limits are defined with respect to the point of interest and the integral over $i$ is trivial if assumed fixed. Given this subset, four possible element sets are found (see Appendix $\underline{\mathrm{C}}$ )

$$
\alpha_{j}^{*}\left(\boldsymbol{r}^{*}, a, e, i\right)=\left(a, e, i, \Omega_{j}, \omega_{j}, f_{j}\right) \quad j=1,2,3,4
$$

Hence, Eq. (71) simplifies to

$$
n_{\boldsymbol{r}}\left(\boldsymbol{r}^{*}\right)=\int_{\frac{r^{*}+R}{2}}^{\infty} \int_{\left|1-\frac{r^{*}}{a}\right|}^{\left|1-\frac{R}{a}\right|} \int_{|\theta|}^{\pi-|\theta|} \sum_{j=1}^{4} \frac{n_{\alpha}\left(\boldsymbol{\alpha}_{j}^{*}\right)}{\left|\operatorname{det} \boldsymbol{J}_{j}^{*}\right|} \mathrm{d} i \mathrm{~d} e \mathrm{~d} a
$$

where the Jacobian $\boldsymbol{J}^{*}$ relates $\boldsymbol{r}^{*}$ to $\left(\Omega^{*}, \omega^{*}, f^{*}\right)$ as

$$
\boldsymbol{J}^{*}=\left.\left(\begin{array}{lll}
\frac{\partial r_{x}}{\partial \Omega} & \frac{\partial r_{x}}{\partial \omega} & \frac{\partial r_{x}}{\partial f} \\
\frac{\partial r_{y}}{\partial \Omega} & \frac{\partial r_{y}}{\partial \omega} & \frac{\partial r_{y}}{\partial f} \\
\frac{\partial r_{z}}{\partial \Omega} & \frac{\partial r_{z}}{\partial \omega} & \frac{\partial r_{z}}{\partial f}
\end{array}\right)\right|_{\boldsymbol{\alpha}=\boldsymbol{\alpha}^{*}}
$$

After some derivations, the determinate is found as

$$
\operatorname{det} \boldsymbol{J}^{*}=\frac{a^{3}\left(1-e^{2}\right)^{3} \cos \left(\omega^{*}+f^{*}\right) \sin f^{*} \sin i}{\left(1+e \cos f^{*}\right)^{4}}
$$

Equation (73) converts a phase space density given in Keplerian elements directly into a spatial density. As before, the volume integral simplifies to an area integral if the inclination of the fragments in the cloud is fixed.

\section{Impact Rate}

Given the phase space density in Cartesian coordinates, the impact rate $\dot{\eta}$ on a target with $\left(\boldsymbol{r}^{*}, \boldsymbol{v}^{*}\right)$ can accurately be estimated as

$$
\dot{\eta}\left(\boldsymbol{r}^{*}, \boldsymbol{v}^{*}\right)=\int_{\mathbb{R}^{3}} A_{c}\left(\frac{\boldsymbol{v}-\boldsymbol{v}^{*}}{\left\|\boldsymbol{v}-\boldsymbol{v}^{*}\right\|}\right) n_{\boldsymbol{r}, \boldsymbol{v}}\left(\boldsymbol{r}^{*}, \boldsymbol{v}\right)\left\|\boldsymbol{v}-\boldsymbol{v}^{*}\right\| \mathrm{d}^{3} \boldsymbol{v}
$$

with the cross-sectional area $A_{c}$ exposed to the incoming flux. Equation (76) is generally valid, without any assumptions on the geometries of chaser or target orbit and includes the possibility to consider the spacecraft orientation. The only assumptions are that the area of the fragments is small compared with the area of the target, and that the flux is constant over $A_{c}$. The first assumption is valid, as $\geq 97.5 \%$ of all fragments are smaller than $L=1 \mathrm{~cm}$. Given the large variance of fragment velocities, the second assumption is reasonable too, especially compared with a default cube size of $10 \mathrm{~km} \times 10 \mathrm{~km} \times$ $10 \mathrm{~km}$ in the CUBE algorithm [27]. As before, $\dot{\eta}$ can be estimated through integration directly in the Keplerian space. The number of impacts, $\eta$, can be calculated by integrating Eq. (76) over time $t$ :

$$
\eta(t)=\int_{t_{0}}^{t} \dot{\eta}\left(\boldsymbol{r}^{*}(t), \boldsymbol{v}^{*}(t)\right) \mathrm{d} t
$$


Alternatively, the average number of impact rate, $\dot{\bar{\eta}}$, over the course of one target orbit might be of interest and can be found as

$$
\begin{aligned}
\dot{\bar{\eta}} & =\frac{1}{P} \int_{0}^{P} \dot{\eta}\left(\boldsymbol{r}^{*}(t), \boldsymbol{v}^{*}(t)\right) \mathrm{d} t \\
& =\frac{1}{2 \pi} \int_{0}^{2 \pi} \dot{\eta}\left(\boldsymbol{r}^{*}(M), \boldsymbol{v}^{*}(M)\right) \mathrm{d} M
\end{aligned}
$$

with the orbital period $P$.

Note that assuming $A_{c}$ to be constant in any direction, i.e., having a spherical target, and given

$$
n_{r, v}=p_{v \mid r} n_{r}
$$

where $p_{v \mid r}$ is the conditional distribution of $\boldsymbol{v}$ given $\boldsymbol{r}$ and $n_{\boldsymbol{r}}$ is the spatial density that can be inferred from Eq. (69), Eq. (76) can be reduced to

$$
\dot{\eta}=A_{c} n_{r} \Delta \bar{v}
$$

where

$$
\Delta \bar{v}=\int_{\mathbb{R}^{3}}\left\|\boldsymbol{v}-\boldsymbol{v}^{*}\right\| p_{\boldsymbol{v} \mid \boldsymbol{r}} \mathrm{d}^{3} \boldsymbol{v}
$$

is the average collision velocity. Equation (80) is equivalent to the time derivative of Eq. (A2). Still, Eq. (76) is preferred to calculate $\dot{\eta}$ because it requires only one integration and $A_{c}$ is not required to be constant in any direction.

\section{Application of the Framework to the Cosmos-2251 Fragmentation Cloud}

The transformation of the initial fragment distribution into subsets of Keplerian elements is shown on the example of a payload collision. The analytical transformation is compared with the sampling method in terms of accuracy. Finally, the spatial density and the collision risk emanating from the cloud of fragments is estimated assuming randomization.

\section{A. Methodologies}

Here, the transformations based on the method of change of variable or the Dirac generalized function presented in Sec. II are referred to as the analytical methods. Analytical methods provide exact solutions and are scalable into higher dimensions. For comparison in terms of sensitivity and scalability, the analytical transformations are compared with densities derived from sampling. To calculate the phase space density from samples, the individual instances, not the density, are transformed into the target space, where they are binned according to given bin geometries. The density is estimated by dividing the number of samples in a bin, $N_{s}\left(V_{x}\right)$, by the total number of samples, $N_{s}$, and by the bin volume, $V_{x}$

$$
\hat{p}_{x}=\frac{N_{s}\left(V_{x}\right)}{N_{s} V_{x}}
$$

Here, the sampling method stands for two seemingly very different methods: Monte Carlo sampling (e.g., [25]) and methods based on finite differences (e.g., [15]). They both have in common that discretization of the phase space is required to estimate densities, such as the phase space or spatial density. As such, they both suffer from poor sensitivity. First, if the number of samples is too small compared with the number of bins, $N_{b}$, the bin might end up with a small number of samples not well representing the actual distribution. Second, binning spreads out highly concentrated distributions over the full bin volume, leading to inaccurate and averaged results, as will become evident herein. Thus, the ideal bin size is a tradeoff between the sensitivity (many small bins) and the accuracy (enough samples in each bin), while keeping in mind that finite differencing methods suffer from poor scalability in dimensions [44]. Hence, binning requires a careful selection of the bin limits, which is dependent on the underlying distribution. In the simplest case, the bins are equally spaced in each separate dimension. Here, the outer bin limits, the range limits, are chosen such as to contain a considerable fraction $\epsilon$ of the overall distribution. Mathematically speaking, $\epsilon$ is defined as

$$
\epsilon=\frac{\int_{B} p_{x} \mathrm{~d} \boldsymbol{x}}{\int_{-\infty}^{\infty} p_{x} \mathrm{~d} \boldsymbol{x}}=\int_{B} p_{x} \mathrm{~d} \boldsymbol{x}
$$

where $B$ is the domain defined by the limits. The domain is regularly discretized with the equal number of bins in each dimension.

\section{B. Study Case}

In February 2009, the two satellites Cosmos-2251 and Iridium 33 collided, resulting in two fragment clouds with hundreds to thousands of fragments large enough to be detected [45]. Here, the initial Cosmos-2251 fragment cloud is modeled and discussed. Considering perturbations from the oblateness of Earth only, and assuming enough time passed for full randomization in $\Omega, \omega$, and $M$, the collision probability toward selected target orbits is estimated. Note that atmospheric drag, which is not considered, considerably changes the shape of the distribution. However, propagation of the cloud in more realistic dynamics is out of the scope of this work. Thus, the resulting collision probabilities are not accurate. The orbit for the Cosmos-2251 parent object at the epoch of collision, and the target orbits as of beginning of 2020 are given in Table 2 .

The NASA SBM distribution is given as a function of $L, A / m$, and $\Delta v$. Here, only the distribution in $\Delta v=10^{v}$ is used, as the dependency on $A / m$ is independent of the transformation of the phase space and no drag perturbations are considered. To facilitate reproduction of the results, the initial distribution in $v$ is assumed to be normally distributed as

$$
v \sim \mathcal{N}\left(\mu_{v}, \sigma_{v}^{2}\right)
$$

with the mean $\mu_{v}=2.63 \log _{10} \mathrm{~m} / \mathrm{s}$ and variance $\sigma_{v}^{2}=$ $0.48^{2}\left(\log _{10} \mathrm{~m} / \mathrm{s}\right)^{2}$. This comes very close to the actual NASA SBM distribution for a payload collision, considering fragments with a characteristic length between $1 \mathrm{~mm}$ and $1 \mathrm{~m}$ (see Fig. 3). The reason is that small fragments, at around $1 \mathrm{~mm}$, dominate the overall distribution. Note that the resulting distribution gives the likelihood of a fragment to be ejected with a certain impulse, not the fragment density. The latter can be calculated by multiplying the likelihood with the number of fragments, $N$. Given the distribution in Eq. (84), $50 \%$ of the fragments are modeled to be ejected with an impulse

\begin{tabular}{|c|c|c|c|c|c|c|c|c|}
\hline Object & International designator & $a, \mathrm{~km}$ & $e$ & $i, \operatorname{deg}$ & $\Omega, \operatorname{deg}$ & $\omega, \operatorname{deg}$ & $f, \operatorname{deg}$ & $A, \mathrm{~m}^{2}$ \\
\hline Cosmos-2251 & 1993-036A & $7,166.1$ & 0.0016 & 74.04 & 19.5 & 98.7 & 358.6 & - \\
\hline Sentinel-1A & 2014-016A & $7,067.0$ & 0.00014 & 98.18 & -- & - & -- & 23.45 \\
\hline Ariane 5 & $2015-039 C$ & $23,840.0$ & 0.7221 & 5.06 & -- & 131.1 & -- & 42.12 \\
\hline
\end{tabular}
larger than $423 \mathrm{~m} / \mathrm{s}$, and $5 \%$ with an impulse larger than $2652 \mathrm{~m} / \mathrm{s}$.

The transformed distribution is studied in three different target spaces. The distribution $p_{a, e}$, in $a$ and $e$, useful when only the longterm evolution of the cloud is to be studied, is found through integration using the Dirac generalized function described in Sec. II.C. The distribution $p_{a, e, \Omega}$, in $a, e$, and $\Omega$, to study the midterm consequences,

Table 2 Parent orbit before fragmentation, and target orbits as of beginning of 2020 and their cross-sectional areas, extracted from [46,47] 


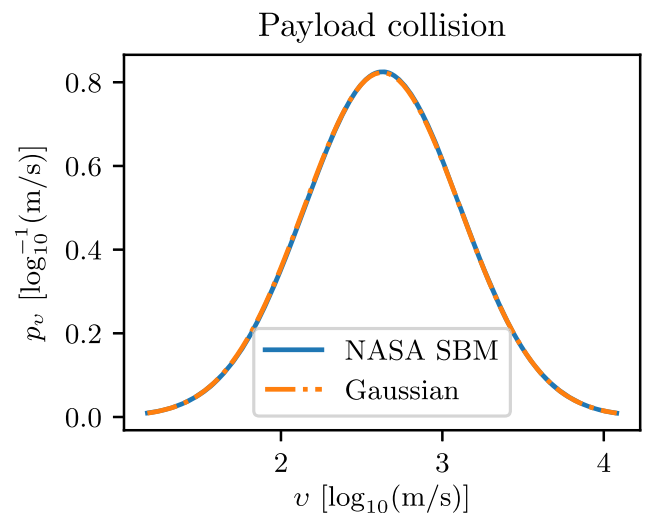

Fig. 3 Integration of the NASA SBM over $L \in[1 \mathrm{~mm}, 1 \mathrm{~m}]$ and $A / m$, and its Gaussian approximation, given as a function of the logarithm to base 10 of $\Delta v$.

is found through the transformation described in Sec. II.D, by using a submatrix of $\boldsymbol{J}_{\alpha s}$ defined by the first, second, and fourth row and the last three columns, relating $\Delta v$ to $(a, e, \Omega)$. For near-circular orbits, or also for eccentric orbits fragmenting close to the apsides, a large part of the transformed distribution is concentrated into a small subspace of the domain. Thus, the distribution in $a, e$, and $\Omega$, is further transformed into $p_{\xi_{p}, \xi_{a}, \Omega}$, in $\xi_{a}, \xi_{p}$, and $\Omega$, with the auxiliary variables defined as

$$
\begin{aligned}
& \xi_{p}=\log _{10}\left(r_{0}-r_{p}\right)=\log _{10}\left(r_{0}-a(1-e)\right) \\
& \xi_{a}=\log _{10}\left(r_{a}-r_{0}\right)=\log _{10}\left(a(1+e)-r_{0}\right)
\end{aligned}
$$

where $r_{0}$ is the radius of fragmentation. The determinate of the Jacobian of the transformation, omitting the one-to-one relationship of $\Omega$, is

$$
\begin{aligned}
\operatorname{det} \boldsymbol{J} & =\operatorname{det}\left(\left[\begin{array}{ll}
\frac{\partial \xi_{p}}{\partial a} & \frac{\partial \xi_{p}}{\partial e} \\
\frac{\partial \xi_{a}}{\partial a} & \frac{\partial \xi_{a}}{\partial e}
\end{array}\right]\right)=\frac{1}{\log ^{2}(10)} \operatorname{det}\left(\left[\begin{array}{cc}
\frac{-1+e}{r_{0}-r_{p}} & \frac{a}{r_{0}-r_{p}} \\
\frac{1+e}{r_{a}-r_{0}} & \frac{a}{r_{a}-r_{0}}
\end{array}\right]\right) \\
& =-\frac{2 a}{\left(r_{0}-r_{p}\right)\left(r_{a}-r_{0}\right) \log ^{2}(10)}
\end{aligned}
$$

The following relationships can be found by taking the exponential of base 10 and summing and subtracting both sides of Eqs. (85):

$$
\begin{aligned}
r_{0}-r_{p} & =10^{\xi_{p}} \\
r_{a}-r_{0} & =10^{\xi_{a}} \\
2 r_{0}-2 a & =10^{\xi_{p}}-10^{\xi_{a}} \\
2 a e & =10^{\xi_{p}}+10^{\xi_{a}}
\end{aligned}
$$

Hence, the distribution function in the new space is obtained according to Eq. (ㅁ) as

$$
\begin{gathered}
p_{\xi_{p}, \xi_{a}, \Omega}=\frac{10^{\xi_{p}+\xi_{a}} \log ^{2}(10)}{2 r_{0}-10^{\xi_{p}}+10^{\xi_{a}}} p_{a, e, \Omega}\left(a=\frac{2 r_{0}-10^{\xi_{p}}+10^{\xi_{a}}}{2},\right. \\
\left.e=\frac{10^{\xi_{p}}+10^{\xi_{a}}}{2 r_{0}-10^{\xi_{p}}+10^{\xi_{a}}}, \Omega=\Omega\right) \quad \forall 10^{\xi_{p}}<r_{0}
\end{gathered}
$$

with the domain restricted to positive values of the perigee radius, i.e., $r_{p}=a(1-e)>0$. The range limits for all three target spaces can be found in Table $\underline{3}$. The defined domains contain $\geq 90 \%$ of the total distribution.
Table 3 Range limits for bin geometries

\begin{tabular}{lcccc}
\hline \hline Space $\boldsymbol{x}$ & $x_{1}$ & $x_{2}$ & $x_{3}$ & $\epsilon$ \\
\hline$(a, e)$ & $4800-17000 \mathrm{~km}$ & $0-0.65$ & -- & $97 \%$ \\
$(a, e, \Omega)$ & $4800-17000 \mathrm{~km}$ & $0-0.65$ & $0-40 \mathrm{deg}$ & $96 \%$ \\
$\left(\xi_{p}, \xi_{a}, \Omega\right)$ & $1.5-6.7 \log _{10}(\mathrm{~m})$ & $1.8-7.3 \log _{10}(\mathrm{~m})$ & $0-40 \mathrm{deg}$ & $94 \mathrm{deg}$
\end{tabular}

The fraction $\epsilon$ describes the share of the distribution located within the limits.

\section{Resulting Fragment Distribution}

\section{Marginalized Target Space}

The fragment cloud distribution in $(a, e)$ is depicted in Fig. 4 for both the sampled and analytically derived transformation. Bins with zero probability, i.e., not affected by the collision or with a lack of samples, are transparent. Large parts of the probability are confined to a small region in the $a-e$ domain (note that the scale is logarithmic). Within just a few bin lengths, the density grows and shrinks by orders of magnitudes. This is a result of both the parent orbit geometry and the log-based breakup model. From the Jacobian in Eq. (19), it becomes evident that, in a first-order approximation, $(\Delta a, \Delta \bar{e})$ are independent of $\Delta v_{h}$. Furthermore, the dependence of $\Delta e$ on $\Delta v_{n}$ nearly vanishes at the point of fragmentation, i.e.,

$$
\begin{aligned}
\left(\begin{array}{c}
\Delta a \\
\Delta e
\end{array}\right) & \approx\left[\begin{array}{cc}
\frac{2 a^{2} v}{\mu} & 0 \\
\frac{2\left(e+c_{f}\right)}{v} & -\frac{r s_{f}}{a v}
\end{array}\right]\left(\begin{array}{c}
\Delta v_{t} \\
\Delta v_{n}
\end{array}\right) \\
& =\left[\begin{array}{cc}
1.92 \times 10^{3} & 0 \\
2.68 \times 10^{-4} & 3.35 \times 10^{-6}
\end{array}\right]\left(\begin{array}{l}
\Delta v_{t} \\
\Delta v_{n}
\end{array}\right)
\end{aligned}
$$

Hence, impulses along the velocity direction have a large effect, dominating the effect of impulses in other directions and producing nearly linearly dependent $(\Delta a, \Delta e)$. Thus, many samples are found near the physical boundary defined by the radius of the parent orbit;

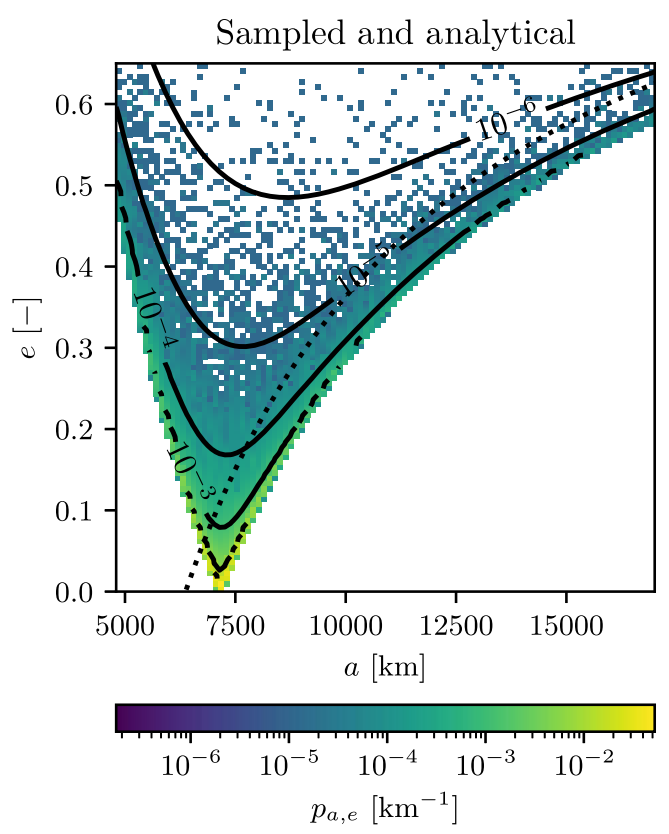

Fig. 4 The marginalized distribution in $(a, e)$ estimated through binning of $N=10^{5}$ samples (color map) and analytical transformation using the Dirac generalized function (contours). The dotted line marks $r_{p}=R$, i.e., fragments with $e>e\left(r_{p}=R\right)$ reenter within a single orbit from the fragmentation. 
e.g., either the perigee or apogee radius of the sample is close the parent orbit radius. As the impulse follows a log-normal distribution, much of the distribution remains close to the origin, whereas the tail end of the distribution produces few fragments that are far away from it. It is noteworthy that large parts of the distribution re-enter directly; i.e., the fragments have a perigee below the radius of Earth, $R=6371 \mathrm{~km}$.

The underlying function poses problems to the sampling method. The physical boundary is not well captured by the bins. Some of the bins extend outward of the boundary spreading probability into a forbidden domain; e.g., fragment probability is added for a perigee above the parent orbit perigee, and vice versa for the apogee, which is physically not feasible. The steep increase and decrease of the underlying distribution lead to order of magnitude jumps in the estimated density. Further from the boundary, where the underlying density becomes small, more and more empty bins can be discerned. A few bins still contain a sample or two, but as a result they overestimate their densities. The analytically derived distribution, instead, exactly represents the underlying distribution in any region and captures the boundary smoothly.

\section{Keplerian Target Space}

The distribution of a fragment in $(a, e, \Omega)$ is shown in Figs. 5 a and $\underline{5 b}$ for both the estimated and analytical solution, for $N=1 \overline{0^{7}}$ samples and $n=100 \times 100 \times 100=10^{6}$ bins. Only three slices are shown in the $\Omega$ direction at $\Omega=10,20$, and $30 \mathrm{deg}$. The color bar is again logarithmic, and zero probability is depicted through transparent bins. The distribution is largely identical to the marginalized distribution, with the major part of the distribution again located near the radial boundary. As small impulses are more likely, the majority remains close to the parent node.

To answer the question about the proper bin size, it is worth investigating the convergence rates at various locations in the domain as the bin volume decreases, i.e., $N_{b} \rightarrow \infty$, and the number of samples increases, i.e., $N_{s} \rightarrow \infty$. The bins for the comparison are defined around a shortlist of $N_{s}=1000$ random samples representing a wide range of density values, equally spaced in the logarithmic weight up to the sample carrying the maximum weight, $p_{a, e, \Omega}^{\max }$. Figure 6 a shows a projection of the samples and the selected bins, with a bin volume that would accommodate $N_{b}=10^{3}$, i.e., 10 bins in each dimension. The diagonal subplots show the samples projected into a single dimension. Off-diagonal subplots show the projection into two dimensions. All the subplots use a logarithmic (color-) scale in order to distinguish features. The "V" shape in the $a-e$ plane can be readily observed. As before, the rectangular bins extend over the physical boundary.
Hence, the estimated value is expected to underestimate the true density, as the bin incorporates a domain of zero probability.

The convergence of the binned distribution as a function of $N_{b}$ and $N_{s}$ is shown in Figs. $\underline{6 b}$ and $\underline{6 c}$ for $N_{s}=10^{9}$ samples and $N_{b}=100^{3}=10^{6}$, respectively. The estimates for bins around small densities converge already with a relatively large bin volume, corresponding to $N_{b} \geq 10^{5}$, well capturing the underlying density. This is because these samples are produced at the tail end of the log-normal distribution where change in the density is slow; i.e., the density is nearly constant over the bin. Bins closer to the peak of the distribution do not converge even for small bins corresponding to $N_{b} \geq 1000^{3}=10^{9}$ because the underlying distribution is too variable. For $N_{b}=100^{3}=10^{6}$, the density around the bin with the maximum density is averaged out to a degree that evaluation of the estimated density underestimates the real density by three orders of magnitude. Any product that is derived from such an averaged density in $(a, e)$, with or without $\Omega$, such as collision probability estimations, will suffer from this inaccuracy. The averaging of the peak over the bin volume will lead to the under- or overestimation of the collision probability, again by orders of magnitude. Hence, for algorithms based on finite differences, the choice of target space is critical.

\section{Bin-Optimized Target Space}

Figure 7 shows the distribution in the $\left(\xi_{p}, \xi_{a}, \Omega\right)$ space. The changes in density to neighboring bins are small compared with the previous space. Additionally, only a small part of the domain describes re-entering fragments, as depicted with the dotted line corresponding to $\xi_{p}\left(r_{p}=R\right)$. Still, large parts of the domain contain empty bins, dotted by nonempty bins with very few samples.

The slow convergence for low-probability events is evident when comparing the densities derived from sampling and analytically in selected bins. Figure 8a shows again $N_{s}=1000$ transformed samples of the distribution and the projections of the 4 bins, with a volume corresponding to $N_{b}=10^{3}$ or 10 bins in each dimension, selected to represent regions of different probabilities. As before, the selected samples are spread equally in the logarithmic density up to the maximum sample density $p_{\xi_{p}, \xi_{a}, \Omega}^{\max }$. The rate of convergence as $N_{b} \rightarrow \infty$ can be seen in Fig. $\underline{8 c}$ for $N_{s}=10^{9}$ samples. As the underlying distribution is not concentrated in small parts of the domain, convergence is achieved for a relatively large bin volume accommodating $N_{b} \geq 10^{5}$ over all regions with large or small probability. Hence, this space is well suited for estimating the underlying distribution function through sampling, as concentrated peaks are nonexistent. Looking at the convergence rate in terms of $N_{s}$ for

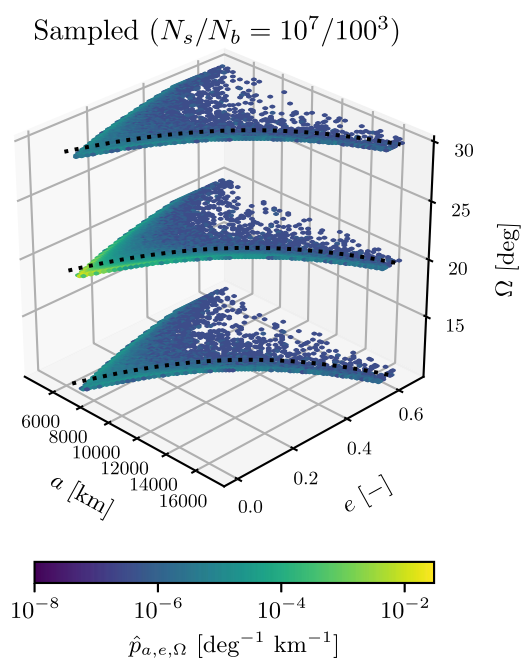

a) The majority of the $N_{s}=10^{7}$ samples end up along the boundary, thus leaving large parts of the domain with empty (transparent) bins

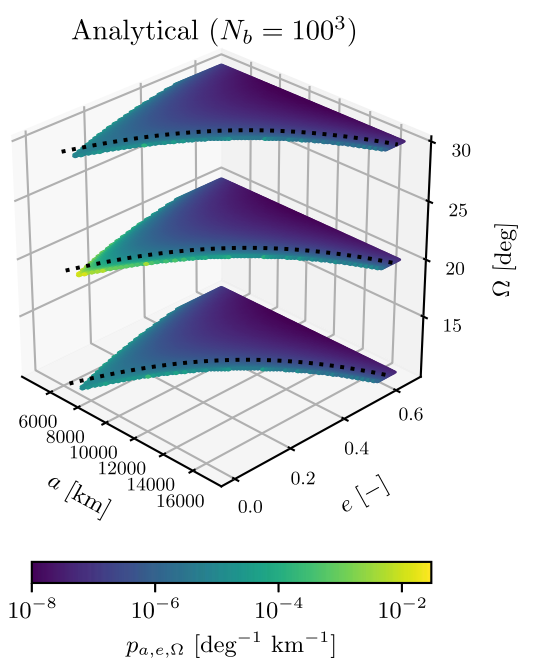

b) The analtyically derived transformation finds a probability even in the domains with near-zero probability, resulting in a high sensitivity

Fig. 5 Comparison between the samples-based and analytically transformed distribution in $(a, e, \Omega)$. Only three slices are shown in $\Omega$ direction. 
Sample distribution $p_{a, e, \Omega}\left[\mathrm{deg}^{-1} \mathrm{~km}^{-1}\right]$
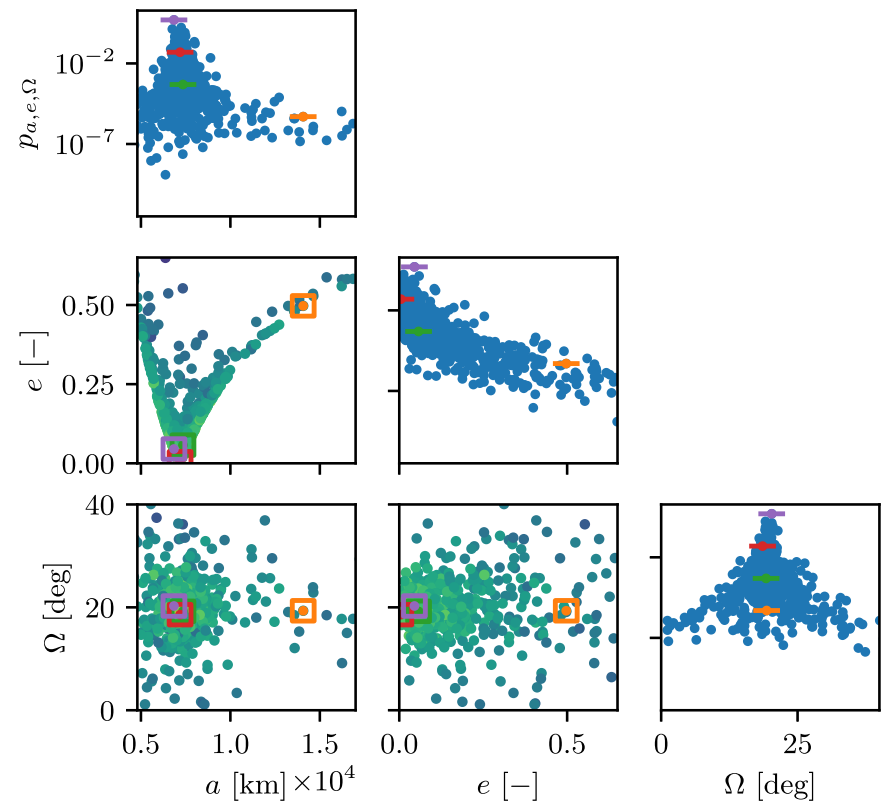

a) Projection of samples and bins chosen for comparison of the two estimation techniques. The represented bin limits show the bin volume accommodating $N_{b}=10^{3}$

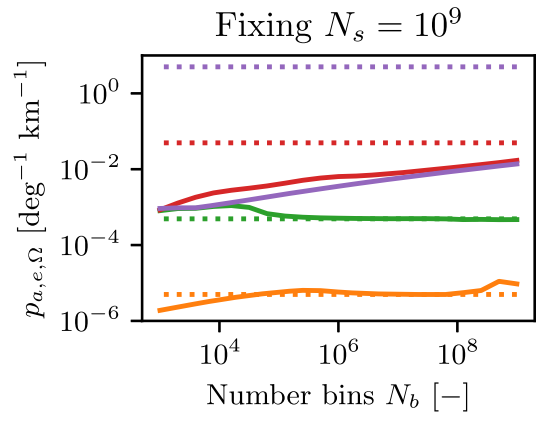

b) The distribution, being unequally distributed, converges only in domains with small densities

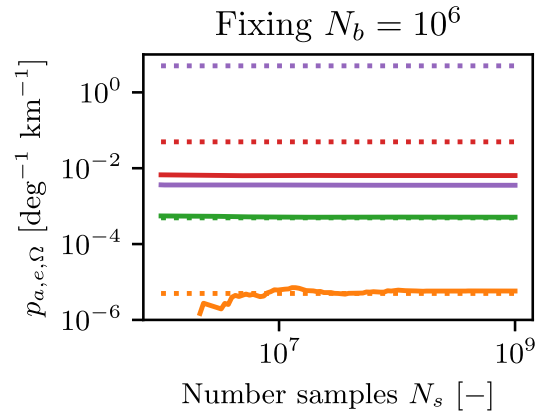

c) Hence, it is not possible to find a single bin volume that fits all the domain

Fig. 6 Distribution and convergence rates of the samples-based method (solid) against the analytical solution (dotted) in $x=(a e \Omega)$.

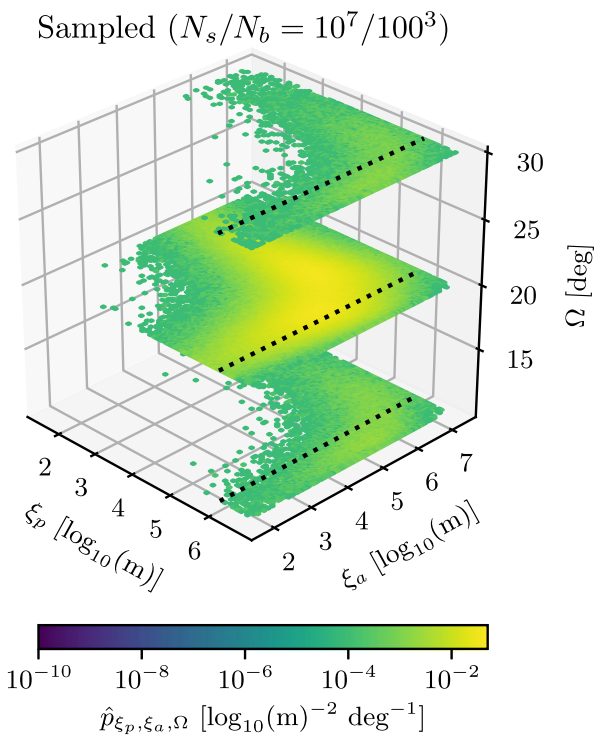

a) The sampling leads to a nonsmooth estimate of the distribution

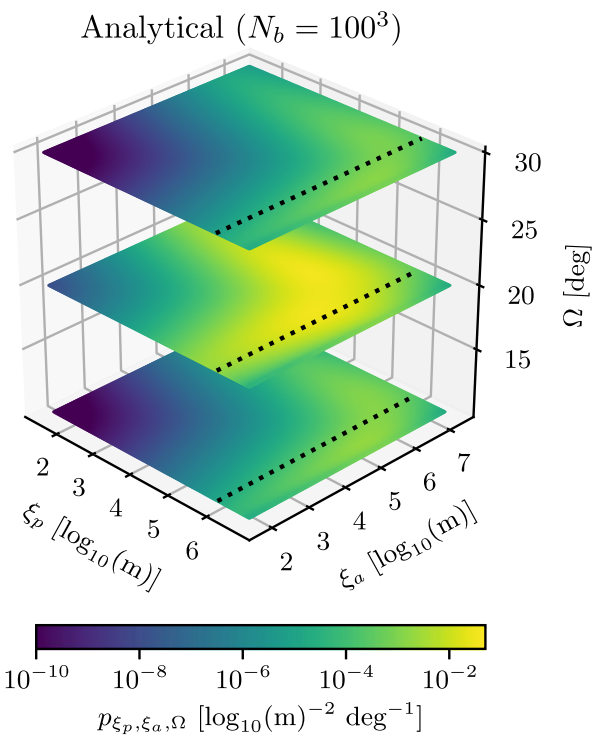

b) Transformed analytically, the distribution can be estimated smoothly

Fig. 7 Comparison between the samples-based and analytically transformed distribution in $\left(\xi_{p}, \xi_{a}, \Omega\right)$. Only three slices are shown in $\Omega$ direction. Fragments with $\xi_{p}>\xi_{p}\left(r_{p}=R\right)$ reenter within a single orbit from the fragmentation. 
Sample distribution $p_{\xi_{p}, \xi_{a}, \Omega}\left[\log _{10}(\mathrm{~m})^{-2} \mathrm{deg}^{-1}\right]$
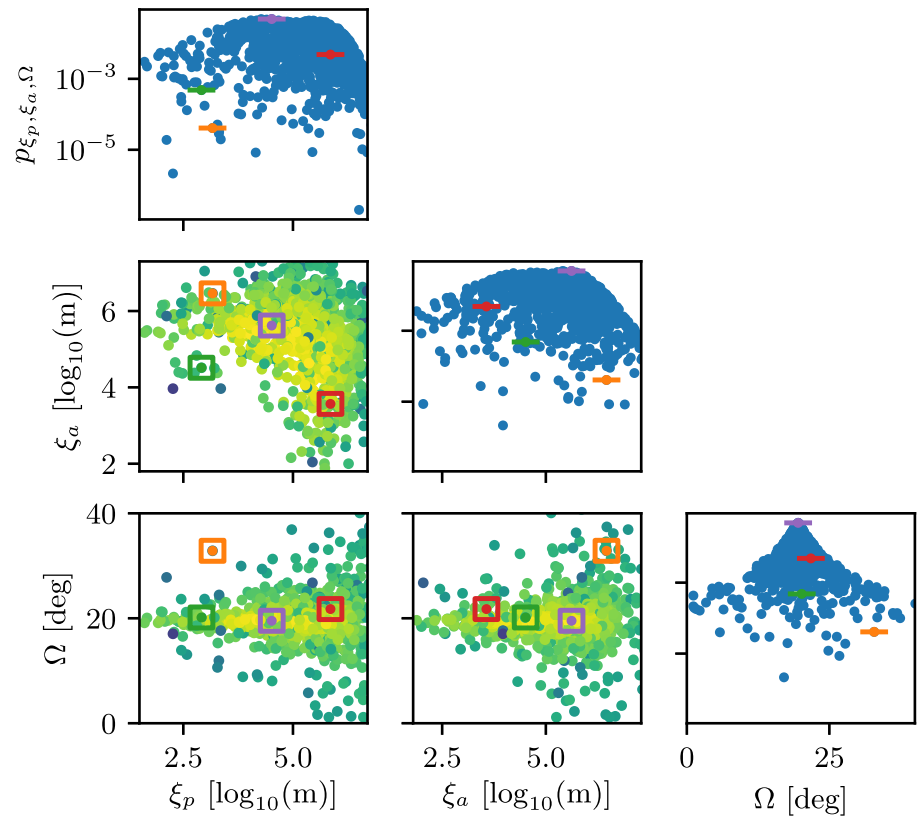

a) Projection of samples and bins chosen for comparison of the two estimation techniques. The represented bin limits show the bin volume accommodating $N_{b}=10^{3}$

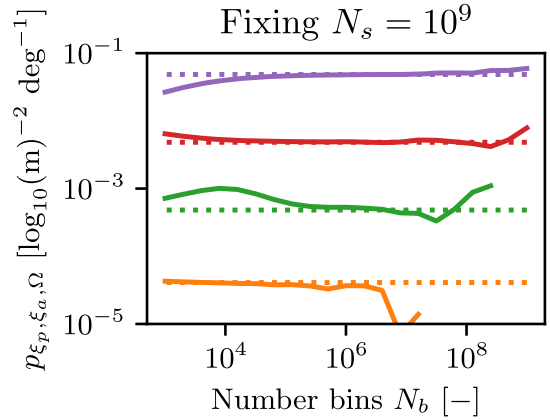

b) The distribution, being smoothly distributed, converges using an $N_{b} \geq 10^{5}$ for all regions

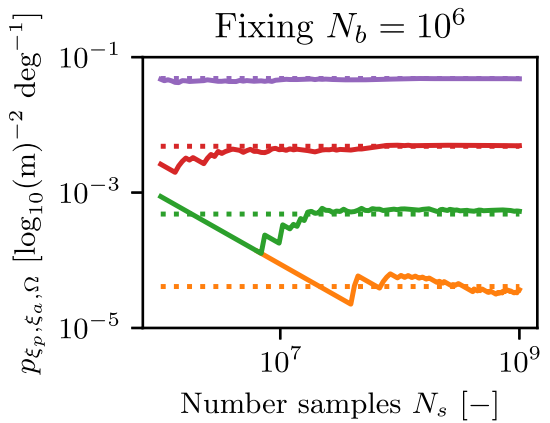

c) However, for $N_{b}=100^{3}$ bins, convergence still requires $N \geq 10^{8}$

Fig. 8 Distribution and convergence rates of the samples-based method (solid) against the analytical solution (dotted) in $x=\left(\xi_{p} \xi_{a} \Omega\right)$.

$N_{b}=10^{6}$ (see Fig. $\underline{8 c}$ ) shows that the estimated densities require more than $N_{s} \geq 10^{8}$ for convergence, showing the lack of sensitivity to capture low probabilities (or rare events) from deterministic samples [29]. Even with large numbers of drawn samples, convergence toward an accurate value of the low density is slow. Extensions to Monte Carlo sampling, such as importance sampling or subset simulation [29], could be used, however, at the expense of global applicability of the method; i.e., each target object would have to be considered individually when calculating the collision probability.

\section{Collision Probability Estimates}

The collision probability is estimated from the analytical, marginalized two-dimensional distribution $p_{a, e}$. Randomization in $\Omega, \omega$, and $M$ is assumed, and a fixed $i=i_{0}$. Hence, the distribution in $\alpha$ and can be obtained as explained in Sec. IV. As before, the densities are transformed into Cartesian coordinates analytically and through sampling. To increase the understanding of the method, the collision probability is derived in a stepwise approach. For the first step, consider a point $\boldsymbol{p}$ in spherical coordinates relative to the inertial frame $\mathcal{I}$

$$
\boldsymbol{p}=\left(\begin{array}{c}
r \\
\lambda \\
\theta
\end{array}\right)=\left(\begin{array}{cc}
7150 \mathrm{~km} \\
0 & \mathrm{deg} \\
0 & \mathrm{deg}
\end{array}\right)
$$

just below the radius of fragmentation, $r_{0}=7154.6 \mathrm{~km}$. The fragments are incident from two planes only, corresponding to the ascending and descending node. The distribution is studied in a frame coplanar to the orbital plane appertaining to the ascending node, which is found through rotation of the inertial frame for $i_{0}$ around the $\hat{\boldsymbol{x}}$ axis. The two coplanar velocity components, $\boldsymbol{v}_{\|}=\left(v_{r} v_{n}\right)$, describe the fragment velocity in $\boldsymbol{p}$ in radial direction and perpendicular to it in the direction of $\boldsymbol{v}$. The out-of-plane velocity $v_{h}$ is always zero. Note that for circular orbits, this frame is identical to the satellite frame $\mathcal{T}$.

The Cartesian distribution $p_{\boldsymbol{r}, \boldsymbol{v}}$ can be found from $p_{\alpha}$ through sampling or analytical transformation. For the sampling, all instances to be found in the bin defined by the ranges

$$
r \in 7150 \mathrm{~km} \pm \frac{\Delta r}{2} \quad \lambda \in \pm 1 \mathrm{deg} \quad \theta \in \pm 1 \mathrm{deg}
$$

are considered, where the $\lambda$ of each fragment needs to be mapped to $\pm 180 \mathrm{deg}$ before the filtering. The resulting distributions for $\Delta r=10 \mathrm{~km}$ and $\Delta r=5 \mathrm{~km}$ are shown in Figs. 9a and 9b. The dotted and the dashed lines mark the minimum $v_{n}=\bar{v}_{n}\left(v_{r}\right)$ required for $r_{p}>R$ and $r_{a}>r_{0}$, respectively. Two observations can be made. A large number of instances are required to obtain enough samples in the volume to describe the velocity distribution. From the originally sampled $N_{s}=2 \times 10^{10}$ instances, only $3.9 \times 10^{4}$ and $1.8 \times 10^{4}$ are 


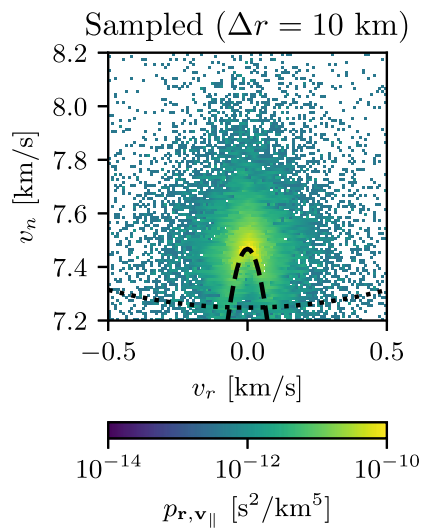

a) If the volume is chosen too large, nonphysical impacts are considered

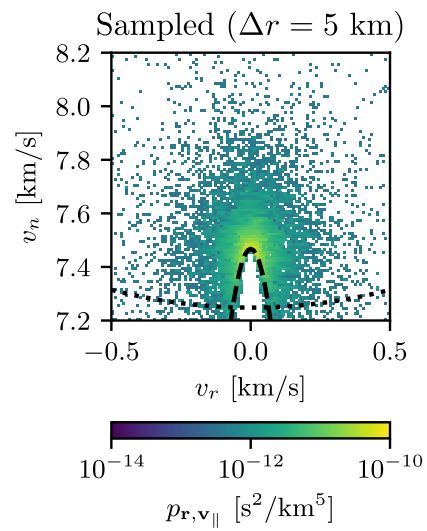

b) If it is chosen too small, only few samples (-) here less than $0.0001 \%$ of $N_{s}(-)$ remain

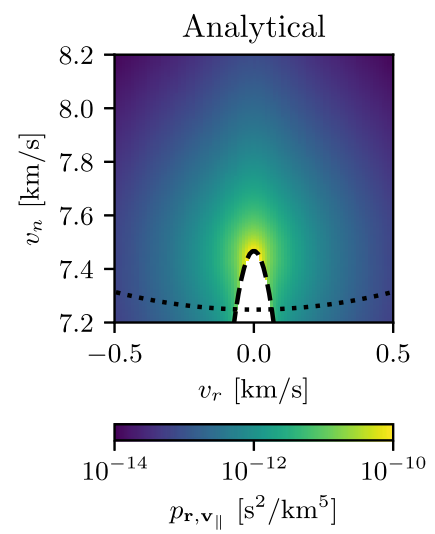

c) The analytical transformation does depend on averaging over a volume

Fig. 9 Incident velocity distribution at chosen point for $N_{s}=2 \times 10^{10}$ samples. The dotted and dashed lines depict the minimum $v_{n}=v_{n}\left(v_{r}\right)$ required for $r_{p}>R$ and $r_{a}>r_{0}$, respectively.

found within the ranges for $\Delta r=10 \mathrm{~km}$ and $\Delta r=5 \mathrm{~km}$, respectively, corresponding to less than $0.0002 \%$ of all the samples. The propagation of this many samples is not feasible. Even if $10^{5}$ samples were to be propagated per second in a parallelized setup, it would still take more than 2 days to propagate all the samples. Hence, Monte Carlo sampling-based methods are not capable of finding such a fivedimensional distribution in a useful time. The second observation is that if the volume is too large, it includes nonphysical impacts. For example, the volume defined by $\Delta r=10 \mathrm{~km}$ extends above $r_{0}$, so it includes samples with $r_{p}>r_{0}$, even though the point of interest, $\boldsymbol{p}$, is nearly $4.6 \mathrm{~km}$ below $r_{0}$. Filtering techniques exist to avoid considering such collisions [48], at the expense of further complicating any deterministic-based method. The analytical transformation is performed by application of Eq. (28), by removing the third row and last column of $\boldsymbol{J}$ corresponding to $i$ and $v_{h}$, respectively. Its distribution is given in Fig. 9c. No integration is required and the resulting distribution is found nearly instantaneously.

The second step is to estimate the spatial density $n_{r}$. Instead of considering only one fragment, the spatial density is estimated considering the total number of fragments, $N$. According to the NASA SBM, the number of fragments in the Cosmos-2251 cloud sized $1 \mathrm{~mm} \leq L \leq 1 \mathrm{~m}$ is $N=2.2 \times 10^{6}$, assuming that $M_{c}=900 \mathrm{~kg}$

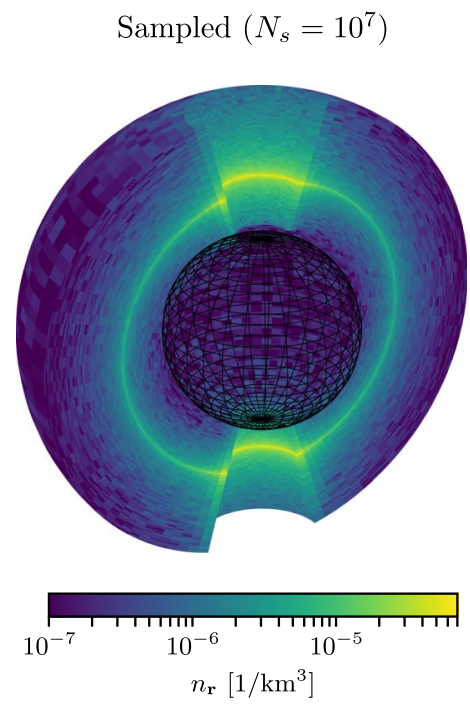

a) Given the large bin volumes, few instances are required to converge to an average over each bin
[49]. For the sampling, a grid in spherical coordinates is defined, splitting the space into bin volumes of 50 equally spaced bins in each direction for

$r \in[R+100 \mathrm{~km}, R+2000 \mathrm{~km}] \quad \lambda \in[0 \mathrm{deg}, 360 \mathrm{deg}]$

$\theta \in\left[\begin{array}{lll}-90 & \mathrm{deg}, 90 \mathrm{deg}\end{array}\right]$

The density obtained through sampling is shown in Fig. 10a for $N_{s}=10^{7}$ instances, showing the spatial density of a single Cosmos-2251 fragment. Given the large bin volumes allows the bin densities to converge quickly with increasing $N_{s}$. However, the distribution is confined to a narrow altitude band, not well captured with the large bins. The analytical spatial density could be obtained by integration of $p_{\boldsymbol{r}, \boldsymbol{v} \|}$ over $v_{r}$ and $v_{n}$. Instead, it is calculated using Eq. (73), for which the integration limits natively ignore re-entered fragments. Its resulting distribution is found in Fig. 10b.

The final step is to calculate the number of impacts and probability of collision for target objects of interest. Because of the large number of instances required to find the velocity distribution in each point, the sampling method is not performed for this derivation. The rate of impact, $\dot{\eta}$, is estimated for Sentinel-1A and the Ariane 5 rocket body

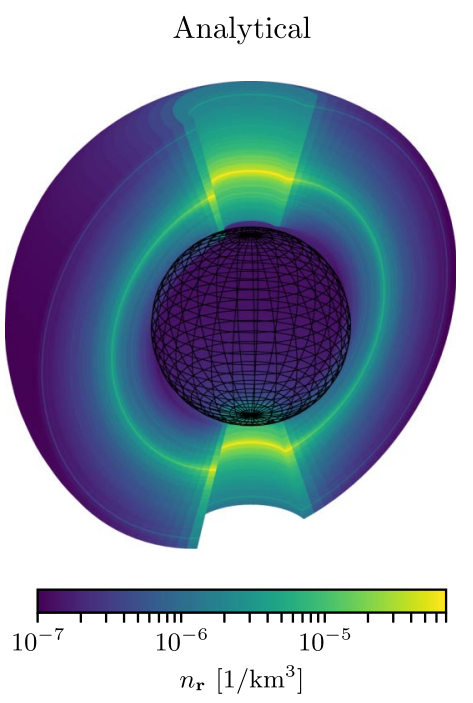

b) As most of the distribution is found in a narrow altitude band, analytical transformation is used to avoid averaging

Fig. 10 Spatial density of the Cosmos-2251 collision fragments, assuming randomization in $\Omega, \omega$, and $M$ (not drawn to scale). 
Impacts

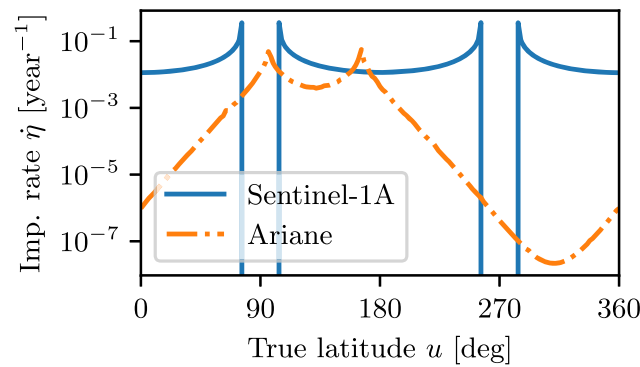

Fig. 11 Impact (Imp.) rates along the orbits of Sentinel-1A and Ariane 5.

according to Eq. (76). Their orbits and cross-sectional areas are reported in Table 2. The resulting impact rate is depicted in Fig. 11. As can be seen, the impact rate is highest when crossing the fragmentation altitude and at high latitudes. The analytical transformation can calculate the impact rate even at GEO altitudes. Averaged over the full target orbit, the fragment impact rate is $\dot{\bar{\eta}}=2.75 \times 10^{-2} \frac{1}{\text { year }}$ for Sentinel-1A and $\dot{\bar{\eta}}=4.48 \times 10^{-4} \frac{1}{\text { year }}$ for the Ariane 5. In terms of collision probability, these impact rates correspond to $p_{1+}=2.7 \%$ and $p_{1+}=0.045 \%$, respectively, if integrated over a year.

\section{Conclusions}

The framework for a fully statistical treatment of the evolution of fragmentation clouds, stemming from explosions or collisions of objects in orbit, and their consequences in terms of collision probability is presented. The NASA SDM is reformulated as a probability distribution function serving as the initial distribution. The discussed transformations enable the propagation of the density in a subset of elements sufficient to describe the mid- to long-term evolution. Furthermore, they are useful in the derivation of the spatial density and the impact rate of the fragments on targets in any orbit. Knowing the impact rate, the collision probability can be obtained. The collision probability can even be calculated, through the randomization of angles, from a distribution defined in few Keplerian elements only. The method is presented on the case of a collision fragmentation cloud and the risk it poses toward a payload in LEO and a rocket body in the geostationary transfer orbit. The sensitivity of the method is high, and it does not suffer from the high computational demand of Monte Carlo-based methods or the inaccuracies of methods based on finite differences and their restriction to low-dimensional problems. The presented framework enables the application of elaborate uncertainty propagation techniques, such as the method of the characteristics, for probabilistic propagation of fragmentation clouds in any dimension and statistical estimation of their ramifications in terms of collision probability with other orbiting objects.

\section{Appendix A: Estimation of Collision Probability}

In their seminal work about the formation of a debris ring around Earth, Kessler and Cour-Palais [16] extend the method developed by Öpik [17] for the estimation of the collision probability from the spatial density. The flux $F$ within a given control volume $\Delta V$ is (analogous to the kinetic gas theory) estimated as [18]

$$
F=n_{r} \Delta \bar{v}
$$

with the fragment spatial density $n_{r}$ assumed constant over $\Delta V$ and the average relative velocity $\Delta \bar{v}$ between the particles in the control volume and a target object of interest. The number of impacts, $\eta$, for an object traversing the volume within $\Delta t$ is then

$$
\eta=F A_{c} \Delta t
$$

where $A_{c}$ is the average collisional cross-sectional area between the fragments and the target. The spatial density in the control volume for a single deterministic object, $n_{r}^{(i)}$, is estimated as [16]

$$
n_{r}^{(i)}=\frac{\Delta t^{(i)}}{P^{(i)}} \frac{1}{\Delta V}
$$

with the time interval $\Delta t$ the fragment spends within $\Delta V$ and the orbital period $P$. The collision rate between two objects in a single volume is then calculated as

$$
\dot{\eta}_{i j}(\Delta V)=n_{\boldsymbol{r}}^{(i)} n_{\boldsymbol{r}}^{(j)} \Delta v_{i j} A_{c} \Delta V
$$

where $\Delta v_{i j}$ is the relative velocity between the two objects. To estimate the total collision rate between two objects, Eq. (A4) is summed over all the volumes accessible to both objects. The spatial density estimate approximated by Eq. (A3) can be further simplified, assuming (just like Öpik [17] and McKnight and Lorenzen [7]) the node and the line of apsides to be fully randomized. Then, the spatial density of an object with given perigee altitude, apogee altitude, and inclination can be found geometrically as a function of radius and latitude only [16].

Finally, the collision probability is found through application of the Poisson function [19]. The Poisson distribution is used to describe, for a specific interval of time, how often an event occurs [20]. Hence, the distribution

$$
p_{k}(\eta)=\frac{\eta^{k} \exp (-\eta)}{k !}
$$

gives a measure of the probability of observing $k$ collisions for a certain time span for which the estimated number of collisions, $\eta$, is valid. The probability of witnessing one or more collision is thus

$$
p_{1+}=1-p_{0}=1-\exp (-\eta)
$$

\section{Appendix B: NASA SBM Parameters}

The parameters presented here are compiled from [24]. Table B1 contains the parameters required to calculate the number of fragments with Eq. (30). The fragmenting mass in the collision, $M_{c}$, is dependent on the impact energy per target mass [ㅁ]

$$
\epsilon=\frac{1}{2} \frac{m_{c}}{m_{t}} v_{c}^{2}
$$

with the mass of the chaser, $m_{c}$, the mass of the target, $m_{t}>m_{c}$, and the impact velocity $v_{c}$. If $\epsilon>40 \mathrm{~J} / \mathrm{g}$, the collision is considered catastrophic; i.e., both chaser and target are completely fragmented, and $M_{c}$ is the sum of both objects:

$$
M_{c}=m_{c}+m_{t}
$$

If $\epsilon>40 \mathrm{~J} / \mathrm{g}$, a noncatastrophic collision occurred; i.e., the target is cratered only. The fragmenting mass is composed of the chaser mass scaled with the square of $v_{c}$, as

$$
M_{c}=m_{c}\left(\frac{v_{c}[\mathrm{~km} / \mathrm{s}]}{1[\mathrm{~km} / \mathrm{s}]}\right)^{2}
$$

Note that this formulation differs from the one given in the original publication; however, it was corrected to be of the form given in Eq. (B3) in [43]. The empirically derived, unitless scaling factor, $0.1 \leq s \leq 1$, is dependent on the explosion body type.

Table B1 Unitless parameters related to the number of fragments, with the mass $M_{c}$ and a scaling factor $s$

\begin{tabular}{lcc}
\hline \hline Fragmentation & $k$ & $\beta$ \\
\hline Collision & $0.1\left(\frac{M_{c}[\mathrm{~kg}]}{1[\mathrm{~kg}]}\right)^{0.75}$ & 1.71 \\
Explosion & $6 \mathrm{~s}$ & 1.6 \\
\hline \hline
\end{tabular}


The parameters required to calculate $\chi$ as a function of $\lambda$ in Eq. (38a) are dependent on the parent type, i.e., rocket body or payload, and on the size regime. The distributions for objects larger than $L>11 \mathrm{~cm}$ were derived by analyzing decay rates of orbital fragments. For both rocket bodies and payloads, the distribution for large objects is bimodal, composed of two normal distributions. For rocket bodies, the parameters for large objects are

$$
\begin{aligned}
& \alpha_{1}= \begin{cases}1 & \lambda \leq-1.4 \\
1-0.3571(\lambda+1.4) & -1.4<\lambda<0 \\
0.5 & 0 \leq \lambda\end{cases} \\
& \mu_{\chi}^{(1)}= \begin{cases}-0.45 & \lambda \leq-0.5 \\
-0.45-0.9(\lambda+0.5) & -0.5<\lambda<0 \\
-0.9 & 0 \leq \lambda\end{cases} \\
& \sigma_{\chi}^{(1)}=0.55 \\
& \alpha_{2}=1-\alpha_{1} \\
& \mu_{\chi}^{(2)}=-0.9 \\
& \sigma_{\chi}^{(2)}= \begin{cases}0.28 & \lambda \leq-1.0 \\
0.28-0.1636(\lambda+1) & -1.0<\lambda<0.1 \\
0.1 & 0.1 \leq \lambda\end{cases}
\end{aligned}
$$

For payloads, the parameters for large objects are

$$
\begin{aligned}
& \alpha_{1}= \begin{cases}0 & \lambda \leq-1.95 \\
0.3+0.4(\lambda+1.2) & -1.95<\lambda<0.55 \\
1 & 0.55 \leq \lambda\end{cases} \\
& \mu_{\chi}^{(1)}= \begin{cases}-0.6 & \lambda \leq-1.1 \\
-0.6-0.318(\lambda+1.1) & -1.1<\lambda<0 \\
-0.95 & 0 \leq \lambda\end{cases} \\
& \sigma_{\chi}^{(1)}= \begin{cases}0.1 & \lambda \leq-1.3 \\
0.1+0.2(\lambda+1.3) & -1.3<\lambda<-0.3 \\
0.3 & -0.3 \leq \lambda\end{cases} \\
& \alpha_{2}=1-\alpha_{1} \\
& \mu_{\chi}^{(2)}= \begin{cases}-1.2 & \lambda \leq-0.7 \\
-1.2-1.333(\lambda+0.7) & -0.7<\lambda<-0.1 \\
-2.0 & -0.1 \leq \lambda\end{cases} \\
& \sigma_{\chi}^{(2)}= \begin{cases}0.5 & \lambda \leq-0.5 \\
0.5-(\lambda+0.5) & -0.5<\lambda<-0.3 \\
0.3 & -0.3 \leq \lambda\end{cases}
\end{aligned}
$$

For small objects $(L<8 \mathrm{~cm}$ ), the distribution (composed of one normal distribution only) is identical for rocket bodies and payloads. The parameters are

$$
\alpha_{1}=1
$$$$
\mu_{\chi}^{(1)}= \begin{cases}-0.3 & \lambda \leq-1.75 \\ -0.3-1.4(\lambda+1.75) & -1.75<\lambda<-1.25 \\ -1.0 & -1.25 \leq \lambda\end{cases}
$$$$
\sigma_{\chi}^{(1)}= \begin{cases}0.2 & \lambda \leq-3.5 \\ 0.2+0.1333(\lambda+3.5) & -3.5 \leq \lambda\end{cases}
$$

\section{Table B2 Parameters for the derivation of the ejection velocity}

\begin{tabular}{lcc}
\hline \hline Type & $\mu_{v}$ & $\sigma_{v}$ \\
\hline Collision & $0.9 \chi+2.9$ & 0.4 \\
Explosion & $0.2 \chi+1.85$ & 0.4 \\
\hline \hline
\end{tabular}

For the range between $8 \mathrm{~cm}<L<11 \mathrm{~cm}$, the original paper simply states that a transition function is being used but does not elaborate on the type of function. For the sake of simplicity, here, a linear transition function in $\lambda$

$$
\alpha_{\lambda}=\frac{\lambda-\lambda_{l}}{\lambda_{u}-\lambda_{l}} \quad \forall \lambda_{l}=\log _{10}(0.08) \leq \lambda \leq \lambda_{u}=\log _{10}(0.11)
$$

is employed, such that

$$
p_{\chi \mid \lambda}^{\text {transition }}=\alpha_{\lambda} p_{\chi \mid \lambda}^{\text {large }}+\left(1-\alpha_{\lambda}\right) p_{\chi \mid \lambda}^{\text {small }} \quad \forall \lambda_{l} \leq \lambda \leq \lambda_{u}
$$

The parameters required to calculate $v$ as a function of $\chi$ in Eq. (38b), depending on the fragmentation type, are given in Table $\overline{\mathrm{B} 2}$. Finally, the parameters required to calculate $A$ as a function of $L$ in Eq. (42a) are

$$
b / \gamma= \begin{cases}0.540424 / 2 & L \leq 0.00167 \mathrm{~m} \\ 0.556945 / 2.0047077 & \text { else }\end{cases}
$$

\section{Appendix C: Intersecting Orbits}

Given a point in space, $\boldsymbol{r}$, that needs to be intersected puts a limit on the Keplerian elements. If the point itself is described through Keplerian elements, the radius $r>0$ can be found via

$$
r=\frac{p}{1+e \cos f}
$$

the latitude, $\theta \in[-\pi / 2, \pi / 2]$, via

$$
\sin \theta=\sin i \sin u
$$

and the longitude, $\lambda \in[0,2 \pi)$, via

$$
\sin (\lambda-\Omega)=\frac{\tan \theta}{\tan i} \cos (\lambda-\Omega)=\frac{\cos u}{\cos \theta}
$$

using any two-argument arctangent routine. The point is then defined as

$$
\boldsymbol{r}=\left(\begin{array}{c}
r \cos \theta \cos \lambda \\
r \cos \theta \sin \lambda \\
r \sin \theta
\end{array}\right)
$$

All the orbits that cross $\boldsymbol{r}$ can be found with simple geometrical considerations. 


\section{C.1. Orbital Planes}

First, note that the inclination needs to be larger than the latitude of the point to be evaluated, resulting in the following range:

$$
i \in\left[i_{0}=|\theta|, i_{1}=\pi-|\theta|\right]
$$

The node $\Omega$ is a function of $\theta, \lambda$, and $i$. From spherical trigonometry, the following dependence can be derived

$$
\sin (\lambda-\Omega)=\frac{\tan \theta}{\tan i}
$$

For each $i$, there exists another orbital plane at $\Omega^{\prime}=\Omega(\lambda, \theta$, $\pi-i)+\pi$ for ascending/descending crossing of $\boldsymbol{r}$. If the point is located over the poles, $\Omega$ is ill-defined.

\section{C.2. Orbit Geometries}

Choosing $a$ and $e$ within the bounds that follow from $R \leq r_{p}=a(1-e) \leq r \leq r_{a}=a(1+e)$

$$
\begin{gathered}
a \in\left[a_{0}=\frac{R+r}{2}\right],\left(a_{1}=\infty\right) \\
e \in\left[e_{0}=\left|1-\frac{r}{a}\right|\right],\left(e_{1}=1-\frac{R}{a}\right)
\end{gathered}
$$

imposes the following rule on $f$

$$
\cos f=\frac{1}{e}\left(\frac{p}{r}-1\right)
$$

To intersect with $r$, the orbit needs to be rotated into the right angle with

$$
\omega=u-f=\sin ^{-1}\left(\frac{\sin \theta}{\sin i}\right)-f
$$

Again, due to symmetry, another $f^{\prime}=2 \pi-f$ exists and thus another $\omega^{\prime}=u-f^{\prime}$.

\section{Acknowledgments}

The research leading to these results has received funding from the European Research Council under the European Unions Horizon 2020 research and innovation program as part of project COMPASS (Grant Agreement No. 679086) and from the European Space Agency through the Networking/Partnering Initiative (NPI) agreement.

\section{References}

[1] Wagner, S., "Maneuver Performance Assessment of the Cassini Spacecraft Through Execution-Error Modeling and Analysis," Proceedings of the 24th AAS/AIAA Space Flight Mechanics Meeting, American Astronautical Soc. Paper 14-390, San Diego, CA, 2014.

[2] Weisman, R. M., Majji, M., and Alfriend, K. T., "Analytic Characterization of Measurement Uncertainty and Initial Orbit Determination on Orbital Element Representations," Celestial Mechanics and Dynamical Astronomy, Vol. 118, No. 2, 2014, pp. 165-195. https://doi.org/10.1007/s10569-013-9529-0

[3] Kminek, G., "ESA Planetary Protection Requirements," European Space Agency Rept. ESSB-ST-U-001, 2012.

[4] Scheeres, D. J., McMahon, J. W., Jones, B. A., and Doostan, A., "Variation of Delivered Impulse as a Function of Asteroid Shape," Proceedings of the IEEE Aerospace Conference, 2015, pp. 1-7. https://doi.org/10.1109/AERO.2015.7119054

[5] Heard, W. B., "Dispersion of Ensembles of Non-Interacting Particles," Astrophysics and Space Science, Vol. 43, No. 1, 1976, pp. 63-82. https://doi.org/10.1007/BF00640556

[6] Jehn, R., "Dispersion of Debris Clouds from On-Orbit Fragmentation Events," ESA Journal, Vol. 15, No. 1, 1991, pp. 63-77.
[7] McKnight, D., and Lorenzen, G., "Collision Matrix for Low Earth Orbit Satellites," Journal of Spacecraft and Rockets, Vol. 26, No. 2, 1989, pp. 90-94. https://doi.org/10.2514/3.26037

[8] McInnes, C. R., "An Analytical Model for the Catastrophic Production of Orbital Debris," ESA Journal, Vol. 17, No. 4, 1991, pp. 293-305.

[9] Letizia, F., Colombo, C., and Lewis, H. G., "Multidimensional Extension of the Continuity Equation Method for Debris Clouds Evolution," Advances in Space Research, Vol. 57, No. 8, 2016, pp. 1624-1640. https://doi.org/10.1016/j.asr.2015.11.035

[10] Wittig, A., Colombo, C., and Armellin, R., "Long-Term Density Evolution Through Semi-Analytical and Differential Algebra Techniques,' Celestial Mechanics and Dynamical Astronomy, Vol. 128, No. 4, 2017, pp. 435-452. https://doi.org/10.1007/s10569-017-9756-x

[11] Frey, S., Colombo, C., and Lemmens, S., "Interpolation and Integration of Phase Space Density for Estimation of Fragmentation Cloud Distribution," Proceedings of the 29th AAS/AIAA Space Flight Mechanics Meeting, American Astronautical Soc. Paper 19-320, San Diego, CA, 2019.

[12] Farinella, P., and Cordelli, A., "The Proliferation of Orbiting Fragments: A Simple Mathematical Model," Science and Global Security, Vol. 2, No. 4, 1991, pp. 365-378. https://doi.org/10.1080/08929889108426373

[13] Talent, D. L., "Analytic Model for Orbital Debris Environmental Management," Journal of Spacecraft and Rockets, Vol. 29, No. 4, 1992, pp. 508-513. https://doi.org/10.2514/3.25493

[14] Smirnov, N. N., Dushin, V. R., Panfilov, I. I., and Lebedev, V. V., "Space Debris Evolution Mathematical Modelling," Proceedings of 1st European Conference on Space Debris, SD-01, European Space Agency, 1993, pp. 309-316.

[15] Letizia, F., "Extension of the Density Approach for Debris Cloud Propagation," Journal of Guidance, Navigation, and Control, Vol. 41, No. 12,2018 , pp. 2650-2656. https://doi.org/10.2514/1.G003675

[16] Kessler, D. J., and Cour-Palais, B. G., "Collision Frequency of Artificial Satellites: The Creation of a Debris Belt," Journal of Geophysical Research, Vol. 83, No. A6, 1978, pp. 2637-2646. https://doi.org/10.1029/JA083iA06p02637

[17] Opik, E. J., "Collision Probabilities with the Planets and the Distribution of Interplanetary Matter," Proceedings of the Royal Irish Academy. Section A: Mathematical and Physical Sciences, Vol. 54, Jan. 1951, pp. 165-199.

[18] Kessler, D. J., "Derivation of the Collision Probability Between Orbiting Objects: The Lifetimes of Jupiter's Outer Moons," Icarus, Vol. 48, No. 1, 1981, pp. 39-48. https://doi.org/10.1016/0019-1035(81)90151-2

[19] Su, S.-Y., and Kessler, D. J., "Contribution of Explosion and Future Collision Fragments to the Orbital Debris Environment," Advances in Space Research, Vol. 5, No. 2, 1985, pp. 25-34. https://doi.org/10.1016/0273-1177(85)90384-9

[20] Soong, T. T., Fundamentals of Probability and Statistics for Engineers, Wiley, Hoboken, NJ, 2004, Chap. 5.

[21] Chobotov, V. A., "Classification of Orbits with Regard to Collision Hazard in Space," Journal of Spacecraft and Rockets, Vol. 20, No. 5, 1983, pp. 484-490. https://doi.org/10.2514/3.25633

[22] McKnight, D., "A Phased Approach to Collision Hazard Analysis," Advances in Space Research, Vol. 10, Nos. 3-4, 1990, pp. 385-388. https://doi.org/10.1016/0273-1177(90)90374-9

[23] Letizia, F., Colombo, C., and Lewis, H. G., "Collision Probability due to Space Debris Clouds Through a Continuum Approach," Journal of Guidance, Control, and Dynamics, Vol. 39, No. 10, 2016, pp. 2240 2249.

https://doi.org/10.2514/1.G001382

[24] Johnson, N. L., Krisko, P. H., Liou, J.-C., and Anz-Meador, P. D., "NASA's New Breakup Model of Evolve 4.0," Advances in Space Research, Vol. 28, No. 9, 2001, pp. 1377-1384. https://doi.org/10.1016/S0273-1177(01)00423-9

[25] Liou, J.-C., Hall, D. T., Krisko, P. H., and Opiela, J. N., "LEGEND-A Three-Dimensional LEO-to-GEO Debris Evolutionary Model," Advances in Space Research, Vol. 34, No. 5, 2004, pp. 981-986. https://doi.org/10.1016/j.asr.2003.02.027

[26] Walker, R., Martin, C. E., Stokes, P. H., Wilkinson, J. E., and Klinkrad, H., "Analysis of the Effectiveness of Space Debris Mitigation Measures Using the DELTA Model," Advances in Space Research, Vol. 28, No. 9, 2001, pp. 1437-1445. https://doi.org/10.1016/S0273-1177(01)00445-8 
[27] Liou, J.-C., "Collision Activities in the Future Orbital Debris Environment," Advances in Space Research, Vol. 38, No. 9, 2006, pp. 2102-2106.

https://doi.org/10.1016/j.asr.2005.06.021

[28] Lewis, H., Diserens, S., Maclay, T., and Sheehan, J. P., "Limitations of the Cube Method for Assessing Large Constellations," Proceedings of the 1st International Orbital Debris Conference, NASA Johnson Space Center, 2019, Paper \#6104.

[29] Au, S.-K., and Beck, J. L., "Estimation of Small Failure Probabilities in High Dimensions by Subset Simulation," Probabilistic Engineering Mechanics, Vol. 16, No. 4, 2001, pp. 263-277.

[30] Luo, Y. Z., and Yang, Z., "A Review of Uncertainty Propagation in Orbital Mechanics," Progress in Aerospace Sciences, Vol. 89, Feb. 2017, pp. 23-39. https://doi.org/10.1016/j.paerosci.2016.12.002

[31] Izzo, D., and Valente, C., "A Mathematical Model Representing the Statistical Properties of Sets of Orbits," Acta Astronautica, Vol. 54, No. 8, 2004, pp. 541-546. https://doi.org/10.1016/S0094-5765(03)00224-8

[32] Izzo, D., "Effects of Orbital Parameter Uncertainties," Journal of Guidance, Control, and Dynamics, Vol. 28, No. 2, 2005, pp. 298-305. https://doi.org/10.2514/1.5650

[33] Vallado, A. V., Fundamentals of Astrodynamics and Applications, 4th ed., Microcosm Press, Cleveland, OH, 2013, Chap. 2.

[34] Au, C., and Tam, J., "Transforming Variables Using the Dirac Generalized Function," American Statistician, Vol. 53, No. 3, 1999, pp. 270-272. https://doi.org/10.2307/2686109

[35] Federer, H., Geometric Measure Theory, Springer, Berlin, 1996, Chap. 3.

[36] Battin, R., An Introduction to the Mathematics and Methods of Astrodynamics, Rev. ed., AIAA, Reston, VA, 1999, Chap. 5.

[37] Vallado, D. A., and Alfano, S., "Updated Analytical Partials for Covariance Transformations and Optimization," Proceedings of the 2015 AAS/ AIAA Astrodynamics Specialist Conference, American Astronautical Soc. Paper 15-537, San Diego, CA, 2015.

[38] Gonzalo, J. L., Colombo, C., and Di Lizia, P., "Analytical Framework for Space Debris Collision Avoidance Maneuver Design," Journal of Guidance, Navigation, and Control, 2019.

[39] Danielson, D. A., Sagovac, C. P., Neta, B., and Early, L. W., "Semianalytic Satellite Theory," Naval Postgraduate School TR ADA276836, Monterey, CA, 1995.
[40] Brouwer, D., "Solution of the Problem of Artificial Satellite Theory Without Drag," Astronomical Journal, Vol. 64, No. 1274, 1959, pp. 378-396. https://doi.org/10.1086/107958

[41] Schaub, H., Vadali, S. R., Junkins, J. L., and Alfriend, K. T., "Spacecraft Formation Flying Control Using Mean Orbit Elements," Journal of the Astronautical Sciences, Vol. 48, No. 1, 2000, pp. 69-87.

[42] Finkleman, D., Oltrogge, D., Faulds, A., and Gerber, J., "Analysis of the Response of a Space Surveillance Network to Orbital Debris Events," Proceedings of the AAS/AIAA Astrodynamics Specialist Conference, American Astronautical Soc. Paper 08-127, San Diego, CA, 2008.

[43] Krisko, P. H., "Proper Implementation of the 1998 NASA Breakup Model," Orbital Debris Quarterly News, Vol. 15, No. 4, 2011 , pp. 1-10.

[44] Sun, Y., and Kumar, M., "A Markov Chain Monte Carlo Particle Solution of the Initial Uncertainty Propagation Problem," Proceedings of the AIAA Guidance, Navigation, and Control Conference, AIAA Paper 2012-4937, 2012. https://doi.org/10.2514/6.2012-4937

[45] Liou, J. C., and Shoots, D., "Satellite Collision Leaves Significant Debris Clouds," Orbital Debris Quarterly News, Vol. 13, No. 2, 2009. p. 1.

[46] "Historical TLE Search Provides Orbital States of Spacecraft and Other Objects," Space-Track, 2020, https://www.space-track.org/.

[47] "DISCOSweb: A Web Based Frontend to ESA's DISCOS," European Space Agency, 2020, https://www.nature.com/nature/ [retrieved Feb. 2020].

[48] Hoots, F. R., Crawford, L. L., and Roehrich, R. L., "A Description of Filters for Minimizing the Time Required for Orbital Conjunction Computations," Celestial Mechanics, Vol. 33, No. 2, 1984, pp. 143 158. https://doi.org/10.1007/BF01234152

[49] Kelso, T. S., "Analysis of the Iridium 33-Cosmos 2251 Collision," Proceedings of the AAS/AIAA Astrodynamics Specialist Conference, American Astronautical Soc. Paper 09-368, San Diego, CA, 2009.

[50] Krisko, P. H., "The Predicted Growth of the Low-Earth Orbit Space Debris Environment-An Assessment of Future Risk for Spacecraft," Proceedings of the Institution of Mechanical Engineers, Part G: Journal of Aerospace Engineering, Vol. 221, No. 6, 2007, pp. $975-985$. https://doi.org/10.1243/09544100JAERO192 